\title{
Observations of 3-D Transverse Dispersion and Dilution in Natural Consolidated Rock by X-Ray Tomography
}

\author{
Maartje Boon ${ }^{\mathrm{a}, \mathrm{b}, *}$, Branko Bijeljic ${ }^{\mathrm{a}, \mathrm{b}}$, Ben Niu ${ }^{\mathrm{a}, \mathrm{b}}$, Sam Krevor ${ }^{\mathrm{a}, \mathrm{b}}$ \\ ${ }^{a}$ Department of Earth Science and Engineering, South Kensington Campus, Imperial \\ College London, SW7 2AZ, United Kingdom \\ ${ }^{b}$ Qatar Carbonates and Carbon Storage Research Centre, Imperial College London
}

\begin{abstract}
Recent studies have demonstrated the importance of transverse dispersion for dilution and mixing of solutes but most observations have remained limited to two-dimensional sand-box models. We present a new core-flood test to characterize solute transport in 3-D natural-rock media. A device consisting of three annular regions was used for fluid injection into a cylindrical rock core. Pure water was injected into the center and outer region and a $\mathrm{NaI}$ solution into the middle region. Steady state transverse dispersion of $\mathrm{NaI}$ was visualized with an X-ray medical CT-scanner for a range of Peclét numbers. Three methods were used to calculate $D_{t}$ : (1) fitting an analytical solution, (2) analyzing the second-central moment, and (3) analyzing the dilution index and reactor ratio. Transverse dispersion decreased with distance due to flow focusing. Furthermore, $D_{t}$ in the power-law regime showed sub-linear behavior. Overall, the reactor ratios were high confirming the homogeneity of Berea sandstone.
\end{abstract}

Keywords: solute transport, transverse dispersion, dilution index, reactor ratio, rock heterogeneity, X-ray tomography

\footnotetext{
* Corresponding author

Email addresses: m.boon13@imperial.ac.uk (Maartje Boon), b.bijeljic@imperial.ac.uk (Branko Bijeljic), b.niu@imperial.ac.uk (Ben Niu), s.krevor@imperial.ac.uk (Sam Krevor)
} 


\section{Introduction}

Dispersion of solute plumes plays an important role in many scientific and applied fields including groundwater contamination [33, 28, enhanced oil recovery [48] and $\mathrm{CO}_{2}$ sequestration [38, 48, 45].

In the past, longitudinal dispersion, which is typically considerably larger than transverse dispersion, has received most of the attention. Several notable studies, however, have shown that transverse dispersion can not be neglected as it plays a key role in the dilution and mixing of solutes and mixing-limited reactive transport $[14,13,31,15,7,8,16,20$. The main goal of our study is to devise an experimental technique capable of quantifying three-dimensional transverse dispersion and dilution in natural porous media by providing several quantities such as the transverse dispersion coefficient [41, the dilution index, and the reactor ratio $[29$.

Transverse dispersion is the combined effect of transverse mechanical spreading 15 due to small scale velocity variations in the advective flux and molecular diffusion. This makes it highly dependent on transport conditions characterized by the Peclét number $(\mathrm{Pe})$. Pe is the ratio between the time needed for solutes to travel a characteristic length $L$ by diffusion $t_{d i f}$, and the time needed for solutes to travel the same length by advection $t_{a d v}$ :

$$
P e=\frac{t_{d i f}}{t_{a d v}}=\frac{\frac{L^{2}}{D_{m}}}{\frac{L}{v}}=\frac{L v}{D_{m}},
$$

${ }_{20}$ where $D_{m}$ is the molecular diffusion coefficient $\left[\mathrm{m}^{2} / \mathrm{s}\right]$ and $v$ the average fluid velocity $[\mathrm{m} / \mathrm{s}]$ obtained by $Q /(A \times \phi)$ where $Q$ is volumetric flow rate $\left[\mathrm{m}^{3} / \mathrm{s}\right], A$ is the cross-sectional area $\left[\mathrm{m}^{2}\right]$, and $\phi$ is porosity $[3]$.

For laminar flow, the dependence of transverse dispersion on Pe can be divided into four different regimes [41, 3, 17: (1) restricted diffusion, (2) transition, (3) power-law and (4) mechanical dispersion. The restricted diffusion regime (1) is defined by: 


$$
\frac{D_{t}}{D_{m}}=\frac{1}{F \phi} \quad P e<<1,
$$

in which $F$ is the formation factor. Advection and diffusion have the same order of magnitude contributions to dispersion in the transition regime $(0.3<\mathrm{Pe}<5)$ and this regime is not strictly defined by an equation. The power-law regime is 30 given by

$$
\frac{D_{t}}{D_{m}}=\beta P e^{\delta} \quad 5<P e<300,
$$

in which $\beta$ and $\delta$ are fitting parameters obtained from experimental or model results. The mechanical dispersion regime is defined by

$$
\frac{D_{t}}{D_{m}}=\alpha P e \quad P e>300
$$

in which $\alpha$ is a fitting parameter obtained from experimental or model results.

Sub-linear behavior of $D_{t}$ with Pe (velocity) in the power-law regime has been observed both experimentally [9, 30, 34, and in pore-scale modeling [3, 42] and has been attributed to incomplete mixing at the pore throats [30].

It is common practice to take the transverse dispersion coefficient to be one order of magnitude smaller than the longitudinal dispersion coefficient [1, 23] but unless one studies solute transport in the advective-dominated regime this is not 40 appropriate [3]. As many groundwater flows do not often fall within the purely advective-dominated regime, there is a clear need for accurate experimental studies on transverse dispersion.

Traditionally, dispersion of solutes has been described by the second central moment, with the dispersion coefficient given by

$$
D_{t}=\frac{1}{2} \frac{d \sigma^{2}}{d t} .
$$

${ }_{45}$ For Gaussian plumes, which are fully characterized by their mean position and covariance matrix of displacements, the latter metric would also be indicative 
of solute dilution. When the plume starts to become non-Gaussian, the second central moment can still reasonably describe spreading, (although with limitations [5]) but it does a very poor job in describing dilution. Kitanidis [29] 50 introduced the dilution index, $E(t)$, defined as

$$
E(t)=\Delta V \exp \left(-\sum_{k=1}^{m} P_{k}(t) \ln \left(P_{k}(t)\right)\right)
$$

where $\Delta V$ is the volume of the domain divided by the number of bins used to discretize the spatial domain. $P_{k}(t)$ is the ratio of the solute mass in each bin to the total solute mass in the system at time $t$ and $m$ is the number of bins in the system. The dilution index defines how much volume is occupied by the solute. Of all plumes with the same mass and mean, the Gaussian plume has the highest degree of dilution. The ratio of the dilution index of a plume and its equivalent Gaussian plume can, therefore, be used as a measure of incomplete mixing and is called the reactor ratio [29, 46].

In the laboratory, transverse dispersion coefficients have been determined using four different methods: (1) from transport of volatile compounds across the capillary fringe [30, (2) by pool dissolution experiments [43, 21], (3) by the length of reactive plumes [15, and (4) by tracer tests. Tracer tests are most common and based on three principle techniques: (1) an annular core device with a uniform solute injected into the core region and concentration measurements of the effluent [6, 27, 22, (2) a device equally divided into two sides with a uniform solute injected at one-side and concentration measured using X-ray light attenuation [24], at probe locations along the core [25, 26] or of the effluent [35, and (3) a device for point source injection of a solute of uniform concentration and concentration measurements taken at several points along the center line of the column [39, 32]. Benekos et al. [2] proposed a different technique with a helix and a cochlea device. With the exception of the technique of Benekos et al. 2], tracer tests are sensitive to plume meandering and prone to overestimation of the transverse dispersion.

All mentioned methods estimate a single average transverse dispersion co- 
efficient by fitting the experimental results to analytical solutions or numerical simulations and do not provide any information about the local heterogeneity of the system. Furthermore, most of the experiments were carried out using sand [6, 21, 30, 15, 22] or bead packs [26, 27, 25, 39, 43, 2, 22, besides Grane [24] who determined the transverse dispersion coefficient in a Berea sandstone that 80 is known to have homogeneous flow and transport characteristics [4].

To the best of our knowledge there are no techniques reported in the literature to make controlled observations of transverse dispersion, at high spatial resolution, in three dimensions, in natural consolidated rock. In this work we present a technique using X-ray tomography in combination with a new inlet configuration to observe a steady-state concentration profile in a cylindrical rock core.

The goal of this study is two fold: (1) to obtain high quality spatially resolved data on transverse dispersion for a range of $\mathrm{Pe}$ that allow us to test existing theories of transverse dispersion, and (2) to present a technique that 90 is applicable to natural consolidated rock representative of reservoir rocks. In addition, the new inlet configuration allows us to observe the spatial variation of transverse dispersion which can be used to characterize rock heterogeneity. Furthermore, the dilution index and the reactor ratio can be obtained as an additional metric of transport heterogeneity.

${ }_{95}$ The paper is organized as follows. In Section 2 we describe the experimental apparatus and the procedure. To aid the experimental design and the analysis of the experimental results, we present in Section 3 analytical solutions of the concentration profile under the transport conditions studied, including a general solution following the approach of Chen et al. [10] and a simplified solution ignoring longitudinal dispersion. The transverse dispersion coefficients were derived from observed concentration fields of $\mathrm{NaI}$ solutes at Pe ranging from 0.5 to 100 using three different methods: (1) fitting an analytical solution, (2) analyzing the second central moment and (3) analyzing the dilution index, as described in Section 4 . In Section 5 we first validate the technique by recovering conventional core average transverse dispersion coefficients in a 
Berea sandstone that are shown to be comparable with literature values from observations by Grane [24] and pore-scale simulations of Bijeljic and Blunt [3]. Furthermore, we provide values of the dispersivity, the dilution index, and the reactor ratio and demonstrate how our findings can be used to fully characterize transverse transport behavior in consolidated porous media. Implications of our experimental findings are discussed in Section 6 while conclusions are drawn in Section 7 .

\section{Experimental Apparatus}

Steady-state transport of a NaI aqueous solution continuously injected into 115 a cylindrical Berea sandstone core, $2.00 \times 10^{-1} \mathrm{~m}$ long and $7.62 \times 10^{-2} \mathrm{~m}$ in diameter, was visualized in three dimensions with the use of a medical X-ray CT scanner. The tube current, tube potential and scan time were set to $200 \mathrm{~mA}$, $120 \mathrm{kV}$, and $1 \mathrm{~s}$, respectively. An inlet end cap device consisting of three annular regions was used to create the boundary condition of the NaI concentration at the inlet of the rock core. Deionized water was injected into the center and the outer annular region, whereas an aqueous solution of $\mathrm{NaI}$ was injected in the middle annular region as illustrated in Fig. 1. The concentration of NaI was $30 \mathrm{wt} \%$, except for low Pe $(0.5$ and 2$)$ where it was $15 \mathrm{wt} \%$. The inner $\left(\rho_{1}\right)$ and outer $\left(\rho_{2}\right)$ radius of the middle annular region were $2.31 \times 10^{-2} \mathrm{~m}$ and $2.77 \times 10^{-2} \mathrm{~m}$, respectively.

A steady and uniform flow field was created at the inlet. For the low Pe (0.5 and 2) this was achieved by placing a ceramic porous plate (1 bar air entry pressure) between the inlet end cap and the core. A $3 \times 10^{-2} \mathrm{~m}$ long plastic ring was placed at the outlet to reduce boundary effects at the end of the core.

${ }_{130}$ The rock core was oriented vertically to minimize effects of gravity. The flow direction was from bottom to top. Before saturating the core with pure water, it was flushed with $\mathrm{CO}_{2}$ to ensure no gas bubbles would form. Three pumps were used for injection, two pure water pumps and one tracer pump. A water pump was used to create the confining pressure (Fig. 1). The experiment was 


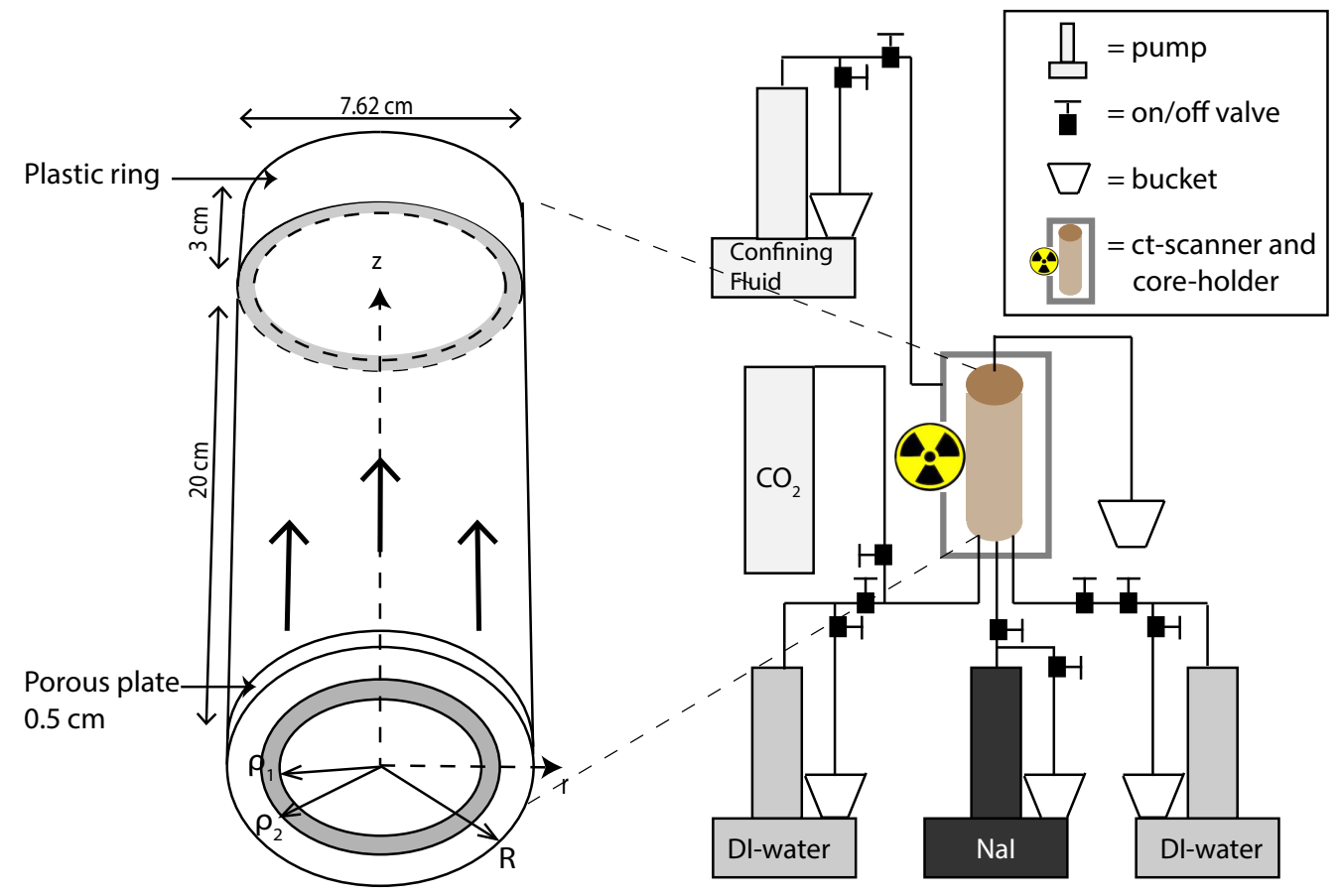

Figure 1: Experimental apparatus - Deionized water is injected in the center and outer annular region. A NaI solution is injected between $\rho_{1}$ and $\rho_{2}$. The big arrows represent the flow direction. 
carried out for $\mathrm{Pe}=0.5,2,10,20,30,40,60$ and 100, representing the restricted diffusion, transition and power-law regimes. The characteristic length in Pe is taken as the grain size $\left(150 \times 10^{-6} \mathrm{~m}\right)$ [37] and the molecular diffusion coefficient $\left(D_{m}\right)$ of $\mathrm{NaI}$ is $1.33 \times 10^{-9} \mathrm{~m}^{2} / \mathrm{s}$. At steady state, X-ray scans were taken every $2 \times 10^{-3} \mathrm{~m}$ along the core. The medical X-ray CT scanner can image at resolutions of $1 \mathrm{~mm}^{3}$ but a significant, random uncertainty might exist in the CT-numbers assigned to each voxel. This uncertainty can be reduced by averaging over multiple images [36].

In this study, images were taken under three different conditions: (1) a core saturated with pure water, (2) a core fully saturated with the NaI-solution, and (3) the core with the annular injection of the NaI-solution at steady state. We calculated the relative concentration of the NaI-solution in the steady state tracer tests by:

$$
\left[C_{N a I}\right]=\frac{C-A}{B-A},
$$

in which $\left[C_{N a I}\right]$ is the relative concentration of the NaI-solution; $\mathrm{A}$ is the voxel CT-number under water-saturated conditions; B is the same number under NaI-

${ }_{50}$ saturated conditions and $\mathrm{C}$ in the steady-state tracer test. All measurements were repeated three times and subsequently averaged to reduce the uncertainty. For the calculation of the transverse dispersion coefficient a coarsening scheme was applied to further reduce the level of noise. For each radial transect, 500 concentration measurements were taken at equal distance. Each concentration measurement was the average concentration within a circle of radius $2.5 \times 10^{-3} \mathrm{~m}$ around the measurement location. This reduced the uncertainty in the concentration measurement to $<5.0 \%$ of the initial concentration.

\section{Mathematical Models}

To aid in the design of the experiments and analyze $D_{t}$, analytical solutions were obtained for transport in a porous medium with a radial geometry and an 
annular source (Fig. 1) similar to the experiments. Axisymmetric conditions were assumed in order to ignore the angular dependence. Two solutions were found for this model: (1) for the two-dimensional advection-dispersion equation (ADE) subject to a third-type inlet boundary condition where the sum of the convective and diffusive fluxes at the inlet are constant and (2) a simplified solution ignoring longitudinal dispersion. For this purpose the approach of Chen et al. 10] was used, modified for the specific geometry of our experiment.

\subsection{Two-Dimensional ADE}

The ADE with uniform coefficients in cylindrical coordinates is given by:

$$
D_{l} \frac{\partial^{2} C}{\partial z^{2}}-v \frac{\partial C}{\partial z}+\frac{D_{t}}{r} \frac{\partial}{\partial r}\left(r \frac{\partial C}{\partial r}\right)=\frac{\partial C}{\partial t},
$$

170

where $C(z, r, t)$ is the solute concentration; $z$ is the coordinate along the core axis; $r$ is the radial coordinate; $t$ is time; $v$ is the average water velocity (interstitial); $D_{l}$ and $D_{t}$ are the longitudinal and transverse dispersion coefficients in the units of $\left[\mathrm{m}^{2} / \mathrm{s}\right]$, respectively. The initial concentration is zero:

$$
C(z, r, t=0)=0 \quad 0 \leq z \leq \infty, \quad 0 \leq r \leq R
$$

where $R$ is the radius of the core.

175 The solute is continuously injected into the middle annular region, which can be expressed by a flux-related boundary condition:

$$
v C(z=0, r, t)-D_{l} \frac{\partial C(z=0, r, t)}{\partial z}= \begin{cases}0 & 0 \leq r \leq \rho_{1} \\ v C_{0} & \rho_{1} \leq r \leq \rho_{2} \\ 0 & \rho_{2} \leq r \leq R\end{cases}
$$

in which $C_{0}$ is the concentration of the injected solute; $\rho_{1}$ is the inner radius of the annular source; $\rho_{2}$ is the outer radius of the annular source. The system is assumed to be of semi-infinite length:

$$
C(z \rightarrow \infty, r, t)=0,
$$


and the mantle of the core acts as no-flux boundary:

$$
\frac{\partial C(z, r=R, t)}{\partial r}=0 .
$$

Eq. 13 gives the steady state solution of the 2-D ADE for the above boundary conditions. The derivation can be found in appendix A, as well as the transient solution that was used to approximate the time needed to reach steady-state in the experiments.

Steady-state solution of the 2-D cylindrical ADE :

$$
\begin{array}{r}
C(r, z, t)= \\
\frac{v C_{0}\left(\rho_{2}^{2}-\rho_{1}^{2}\right)}{R^{2} \sqrt{D_{l}}} \exp \left(\frac{v z}{2 D_{l}}\right) \frac{\exp \left(-\frac{z}{\sqrt{D_{l}}} \sqrt{\frac{v^{2}}{4 D_{l}}}\right)}{\frac{v}{2 \sqrt{D_{l}}}+\sqrt{\frac{v^{2}}{4 D_{l}}}} \\
+\frac{2}{R^{2}} \sum_{n=1}^{\infty} \frac{v C_{0}\left(\frac{\rho_{2}}{\lambda_{n}} J_{1}\left(\lambda_{n} \rho_{2}\right)-\frac{\rho_{1}}{\lambda_{n}} J_{1}\left(\lambda_{n} \rho_{1}\right)\right)}{\sqrt{D_{l}}} \\
\exp \left(\frac{v z}{2 D_{l}}\right) \frac{\exp \left(-\frac{z}{\sqrt{D_{l}}} \sqrt{\frac{v^{2}}{4 D_{l}}+D_{t} \lambda_{n}^{2}}\right)}{\frac{v}{2 \sqrt{D_{l}}}+\sqrt{\frac{v^{2}}{4 D_{l}}+D_{t} \lambda_{n}^{2}}} \frac{J_{0}\left(\lambda_{n} r\right)}{\left|J_{0}\left(\lambda_{n} R\right)\right|^{2}} .
\end{array}
$$

where $\lambda_{n}$ is the Finite Hankel transform parameter which is determined by:

$$
\frac{d J_{0}\left(\lambda_{n} R\right)}{d r}=0
$$

$J_{0}()$ is the zero order Bessel function of the first kind and $J_{1}()$ is the first order Bessel function of the first kind.

\subsection{Two-dimensional cylindrical ADE ignoring longitudinal dispersion}

When longitudinal dispersion is ignored, the 2-D ADE in cylindrical coordi- 


$$
\frac{D_{t}}{r} \frac{\partial}{\partial r}\left(r \frac{\partial C}{\partial r}\right)=v \frac{\partial C}{\partial z} .
$$

At the inlet of the core, the concentration in the middle annular region is $C_{0}$ and zero everywhere else:

$$
C(r, z=0)= \begin{cases}0 & 0 \leq r \leq \rho_{1} \\ C_{0} & \rho_{1} \leq r \leq \rho_{2} \\ 0 & \rho_{2} \leq r \leq R\end{cases}
$$

and no-flux conditions exist at $\mathrm{r}=\mathrm{R}$ :

$$
\frac{\partial C(r=R, z)}{\partial r}=0 .
$$

Eq. 17 gives the analytical solution of the ADE at steady state when longitudinal dispersion is ignored. The derivation can be found in appendix B.

Simplified solution ignoring $D_{l}$ :

$$
\begin{array}{r}
C(r, z)=\frac{C_{0}}{R^{2}}\left(\rho_{2}^{2}-\rho_{1}^{2}\right) \\
+\frac{2}{R^{2}} \sum_{n=1}^{\infty} C_{0}\left(\frac{\rho_{2}}{\lambda_{n}} J_{1}\left(\lambda_{n} \rho_{2}\right)-\frac{\rho_{1}}{\lambda_{n}} J_{1}\left(\lambda_{n} \rho_{1}\right)\right) \exp \left(-D_{t} \lambda_{n}^{2} \frac{z}{v}\right) \frac{J_{0}\left(\lambda_{n} r\right)}{\left|J_{0}\left(\lambda_{n} R\right)\right|^{2}} .
\end{array}
$$

\section{Methods of Analyzing the Experiments}

The radial geometry of the core and annular source used in the experiment (Fig 1) allowed the transverse dispersion coefficients to be calculated using three different methods: (1) fitting the analytical solution (Eq. 17) to the steady state concentration profile, (2) analyzing the second-central moment (Eq. 5) and (3) analyzing the dilution index (Eq. 6).

At steady state, X-ray scans were taken every $0.2 \mathrm{~cm}$ along the core. Fig. 2 shows three of these slices. For each radial transect (the orange lines) the concentration distribution was obtained. From these concentration distributions the transverse dispersion coefficients were calculated. 

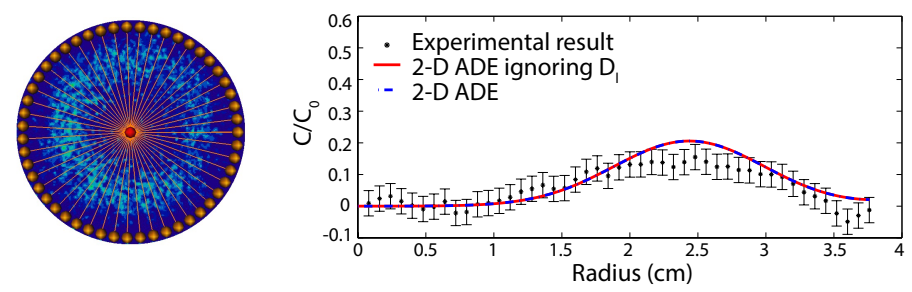

$19 \mathrm{~cm}$ from inlet
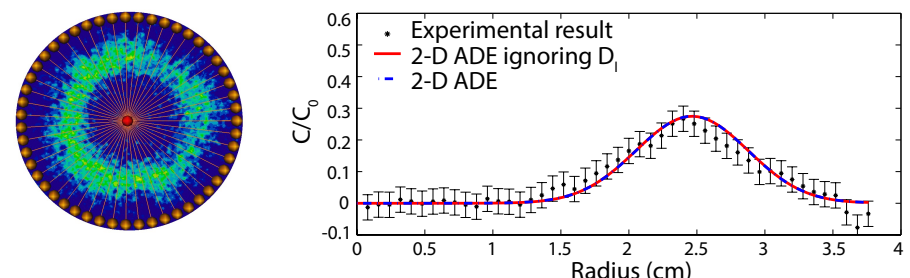

$10 \mathrm{~cm}$ from inlet
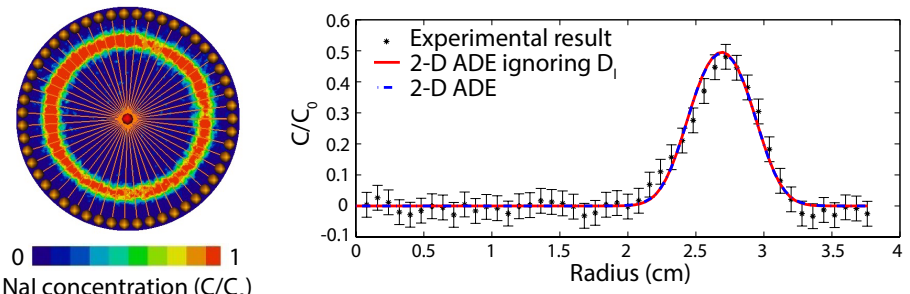

$0.5 \mathrm{~cm}$ from inlet Nal concentration $\left(C / C_{0}\right)$

Figure 2: Left: Three core slices (from bottom to top: inlet, half way, outlet) at steady state for $\mathrm{Pe}=0.5$. The orange lines represent the radial transects. Right: Concentration profiles along one of the radial transects of the core slice. Experimental result: black dots with error bar; 2-D ADE ignoring $D_{l}$ : red solid line; 2-D ADE: blue dot-dash line. 


\subsection{Fitting the Analytical Solution to the Concentration Profile} with the concentration distributions obtained by the analytical solution of the full 2-D ADE (Eq. 13) and the simplified solution ignoring longitudinal dispersion (Eq. 17) for $\mathrm{Pe}=0.5$. The parameter of longitudinal dispersion used in the 2-D ADE is one order of magnitude larger than the transverse dispersion coefficient. It can be seen that the solution of the full 2-D ADE and the simplified solution give almost identical results. Similar results were obtained for higher Pe. Therefore, the transverse dispersion coefficients were obtained by fitting the simplified solution ignoring longitudinal dispersion to the experimental data. For each radial transect at the inlet, the area of $\pi\left(\rho_{2}^{2}-\rho_{1}^{2}\right)$ was calculated. to allow for meandering in the radial direction.

The analytical solution assumes a core of infinite-length. Fig. 3 shows the error in the transverse dispersion coefficient obtained from fitting the analytical solution to the numerical results using the reactive transport code CrunchFlow

230 44 for $\mathrm{Pe}=2$. It can be seen that the error is large at the inlet boundary due to a boundary effect of the numerical model, small in the middle of the core and increases towards the outlet boundary due to the infinite length assumption. Because of the possible existence of experimental boundary effects the last 2 $\mathrm{cm}$ of the core were not used, therefore the error in the calculated transverse

fitting the analytical solution to the numerical result is $1.55 \times 10^{-7} \mathrm{~m}^{2} / \mathrm{s}$, while the actual $D_{t}$ put into the model was $1.58 \times 10^{-7} \mathrm{~m}^{2} / \mathrm{s}$, which is an error of only $1.75 \%$.

\subsection{Second Central Moment}

The transverse dispersion coefficient is defined as the change of the second central moment with time (Eq 5). In the experimental set up of this study, time is the distance along the core $(z)$ divided by the velocity $(v)$ and the transverse dispersion coefficient can be defined as: 


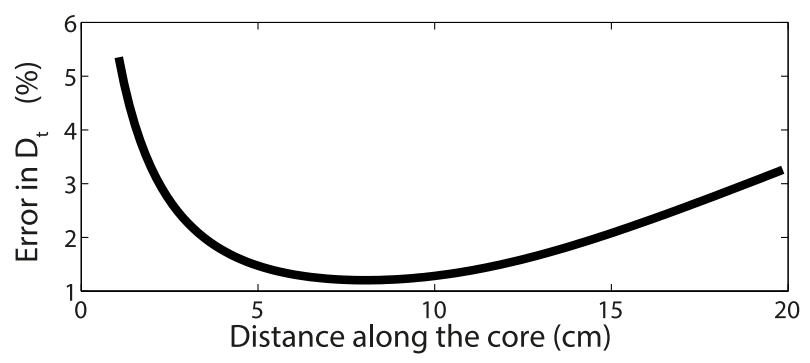

Figure 3: Error in $D_{t}$ from fitting the analytical solution to the numerical CrunchFlow results for $\mathrm{Pe}=2$.

$$
D_{t}=\frac{v}{2} \frac{d \sigma^{2}}{d z} .
$$

It is important to note that this formulation is not derived from the equations and may not work for very long columns, high transverse dispersion coefficients, or a different radial distribution of the solute in the source.

On each of the transects (orange lines on Fig 2) 500 concentration measurements were taken and coarsened into 94 segments, $d r$. Let $\boldsymbol{A}$ and $\boldsymbol{R}$ be vector arrays of the area and coordinates of the center of each radial ring, respectively, and $\boldsymbol{C}$ be a vector array with the solute concentrations of each segment on the transect.

The concentration obtained for each radial segment on the transect was multiplied by the area of that ring to compute the mass of the ring. The sum of the mass of all the rings provides the zero ${ }^{\text {th }}$ radial moment $\left(M_{0}\right)$ :

$$
M_{0}=\sum_{i=1}^{94} A_{i} \times C_{i} .
$$

The first radial moment $\left(M_{1}\right)$ was calculated by multiplying the coordinate of the ring center by the mass of each ring, and then summing over all the rings and dividing it by the total mass: 


$$
M_{1}=\frac{1}{M_{0}} \sum_{i=1}^{94}\left(A_{i} \times C_{i}\right) \times R_{i} .
$$

The second central moment $\left(M_{2}\right)$ was calculated by first calculating the square of the deviation from the mean for each of the locations of the ring centers, and multiplying it by the mass of each ring. Then summing over all the rings and dividing it by the total mass results in:

$$
M_{2}=\frac{1}{M_{0}} \sum_{i=1}^{94}\left(A_{i} \times C_{i}\right) \times\left(R_{i}-M_{1}\right)^{2} .
$$

The transverse dispersion coefficients were calculated from the second central moment by two methods: (1) by fitting the variance length profile obtained with the analytical solution to the experimentally obtained variance length profile averaged over the radial transects, and (2) by plotting the second central moment of each radial transect against distance along the core $(z)$ and fitting a straight line to the data using least squares fitting (Fig. 4). The slope of this line can be used in Eq. 18 to calculate a core average transverse dispersion coefficient.

\subsection{Dilution Index and Reactor Ratio}

For each of the radial transects in Fig. 2 the 1-D dilution index (Eq. 6) was calculated. For this, first $P_{k}(t)$ was obtained. $P_{k}(t)$ is the ratio of the solute mass in each radial segment, to the total solute mass of the radial transect at time $t$. For this purpose, each radial transect was divided in 94 radial segments, $d r$. To account for the radial geometry, the mass of each radial segment was weighted according to the area of the corresponding ring with width $d r$. The total mass of the radial transect $\left(M_{0}\right)$ is the sum of the weighted mass of each of the radial segments. Let $\boldsymbol{A}$ be a vector array of the area of each radial ring and $\boldsymbol{C}$ be a vector array with the solute concentrations of each segment on the transect. $P_{k}(t)$ for each segment of the transect was calculated by dividing the weighted mass of the segment by the total mass of the transect: 

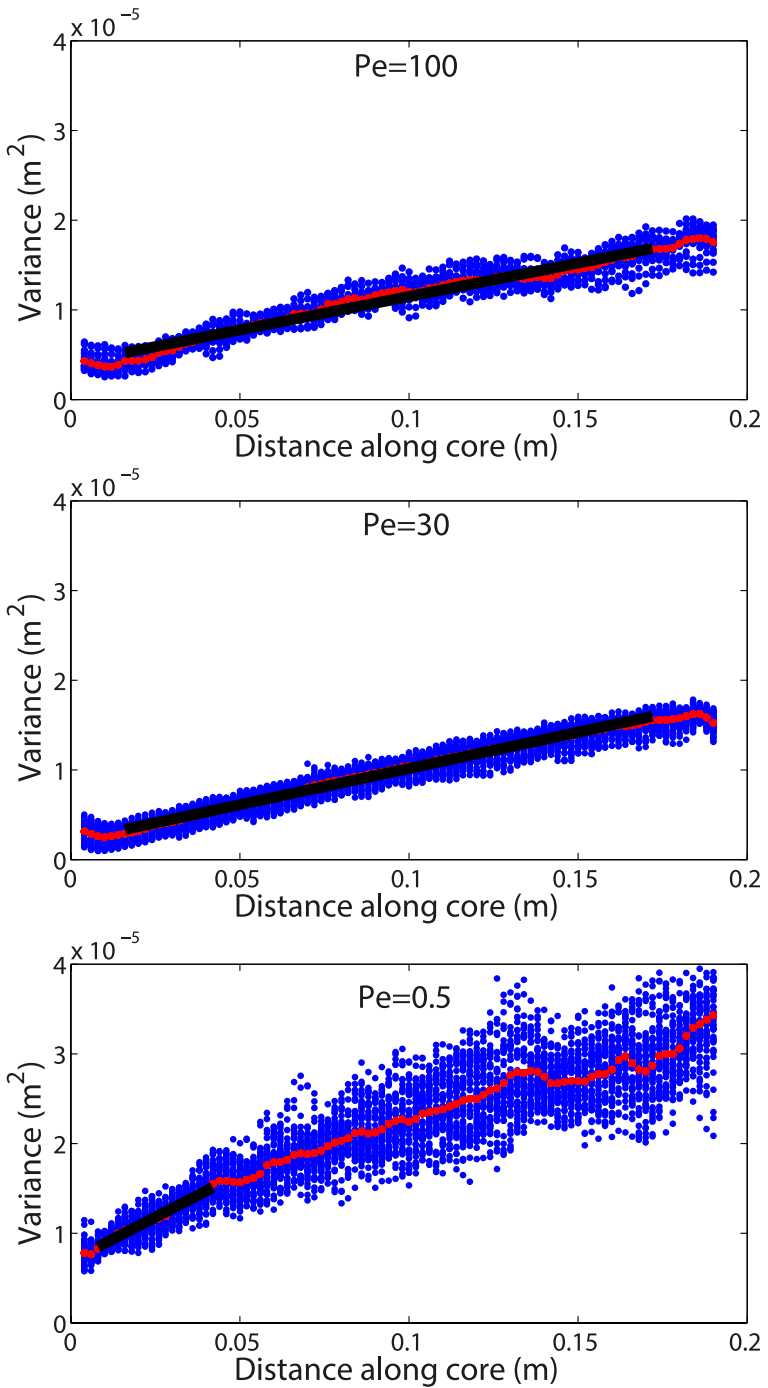

Figure 4: Variance vs distance for $\mathrm{Pe}=0.5, \mathrm{Pe}=30$, and $\mathrm{Pe}=100$. The blue dots show the variance calculated for each of the radial transects (orange lines (Fig. 2). The slope of the black line (fitted to the data points using least squares fitting) is used to calculate $D_{t}$. The running average is shown by the red line. 


$$
P_{k}(t)=A \times C / M_{0}
$$

The dilution index was obtained for each of the 50 transects and 100 slices using Eq. 6 with $\Delta V=d_{r}$.

The transverse dispersion coefficients were calculated from the 1-D dilution index by two methods: (1) by fitting the dilution index length profile obtained with the analytical solution to the experimental dilution index length profile averaged over the radial transects, and (2) from the equation defining the dilution index for 1-D point injection in a homogeneous porous medium (Eq. 23).

Kitanidis 29] showed that the dilution index for instantaneous point in290 jection in a 1-D homogeneous medium, with constant velocity and dispersion coefficient is given by

$$
E(t)=\sqrt{(2 \pi)} \exp (1 / 2) \sqrt{2 D_{t}} \sqrt{t} .
$$

By taking the natural logarithm on both sides, the following expression is obtained:

$$
\ln (E)=\left[\frac{1}{2} \ln (2 \pi)+\frac{1}{2}+\frac{1}{2} \ln \left(2 D_{t}\right)\right]+\frac{1}{2} \ln (t) .
$$

The plot of $\ln (E)$ versus $\ln (t)$ will be a straight line with slope $\frac{1}{2}$ and the dispersion coefficient can be calculated from the intercept.

Because of the radial geometry of the rock and the annular source in this study, axisymmetric conditions can be assumed and each radial transect can be viewed as a 1-D medium with a solute source of finite width $\left(\rho_{2}-\rho_{1}\right)[m]$ at time $t$ where $t=(z / v)$.

300 The transverse dispersion coefficient could therefore be calculated with the use of Eq. 24 for instantaneous point injection with a correction for the finite width of the solute source. This correction was made by adding the time needed for the dilution index to reach $\left(\rho_{2}-\rho_{1}\right)[m]$ to $t_{0}$. To calculate how long it will 
take to spread to this finite width we need to use an estimated value of $D_{t}$. For concentration profile.

Eq. 23 only applies for a homogeneous medium where solute plumes follow Gaussian behavior which corresponds to the highest degree of dilution. In this case the peak solute concentration should decrease with $t^{-1 / 2}$ and should follow 310

The ratio of the dilution index of a plume to its equivalent Gaussian plume is called the reactor ratio:

$$
M_{\text {gaussian }}=\frac{E_{\text {experiment }}}{E_{\text {numerical }}}
$$

The reactor ratio can be used as measure of incomplete plume dilution, with $M \rightarrow 1$ when the plume is very close to a Gaussian plume and $M \rightarrow 0$ when the plume is very irregular and the porous medium is very heterogeneous. In addition, the ratio of the peak concentration to the peak concentration of its Gaussian equivalent can be used [46. To account for the radial geometry used in this study the concentrations were weighted according to the area of the ring $\boldsymbol{A}$.

For the experimental apparatus of this study, the use of the 1-D dilution index is only valid in the case of perfect symmetry. For more heterogeneous cases where extensive plume deformation occurs, the cross-sectional dilution index has to be used instead. The cross-sectional dilution index was obtained for each of the 100 slices using Eq. 6 where $\Delta V$ is the area of the cross-section ratio of the solute mass in each voxel to the total solute mass in the cross-section at time $t$.

\section{Results}

Fig. 5 shows the steady-state concentration profiles, experimentally observed and calculated from the analytical solution $(\mathrm{Eq} 13)$, for the injection of a $\mathrm{NaI}$ 


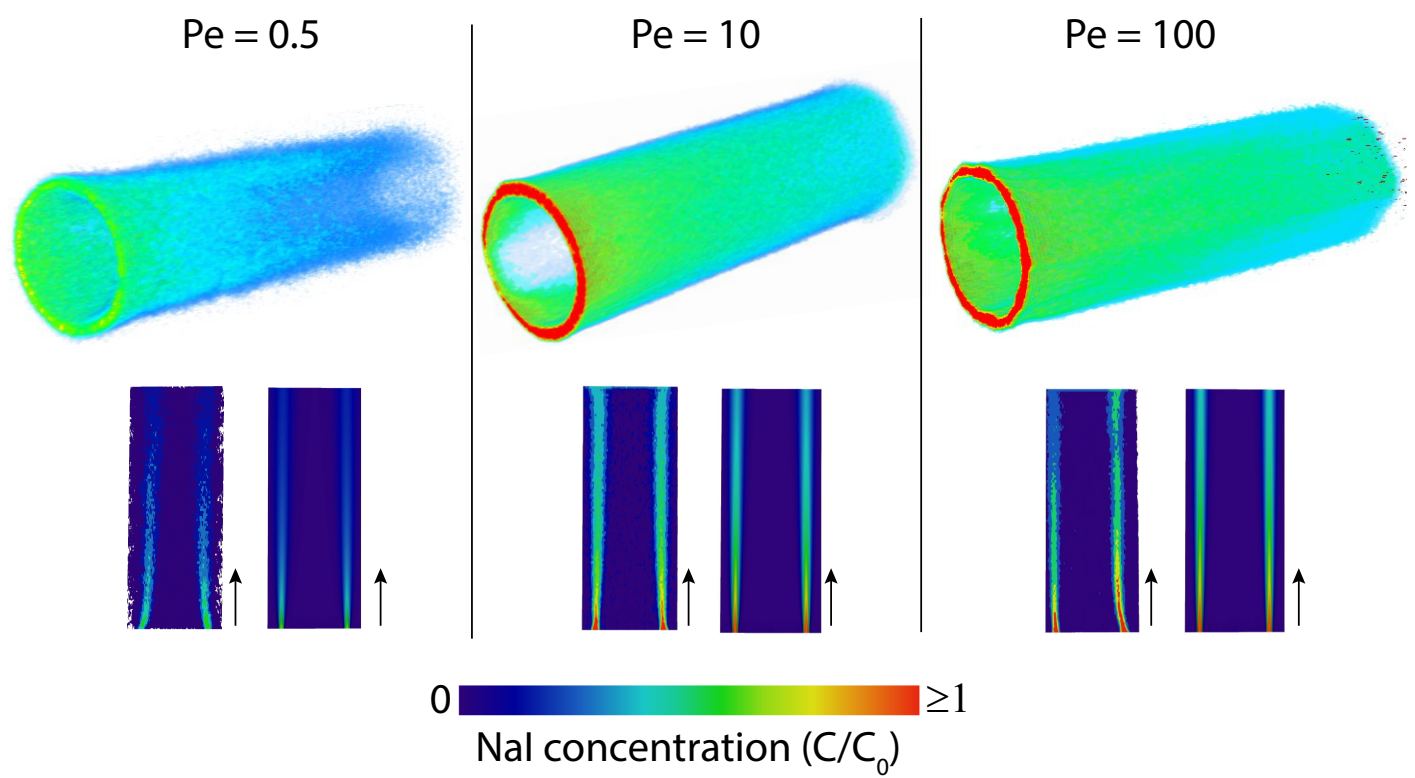

Figure 5: Top: tracer plume at steady state. Bottom: cut-through along the cores axis for the experimental results (left) and the results from the analytical solution (right)) for $\mathrm{Pe}=0.5$, $\mathrm{Pe}=10$ and $\mathrm{Pe}=100$. The arrows represent flow direction. 


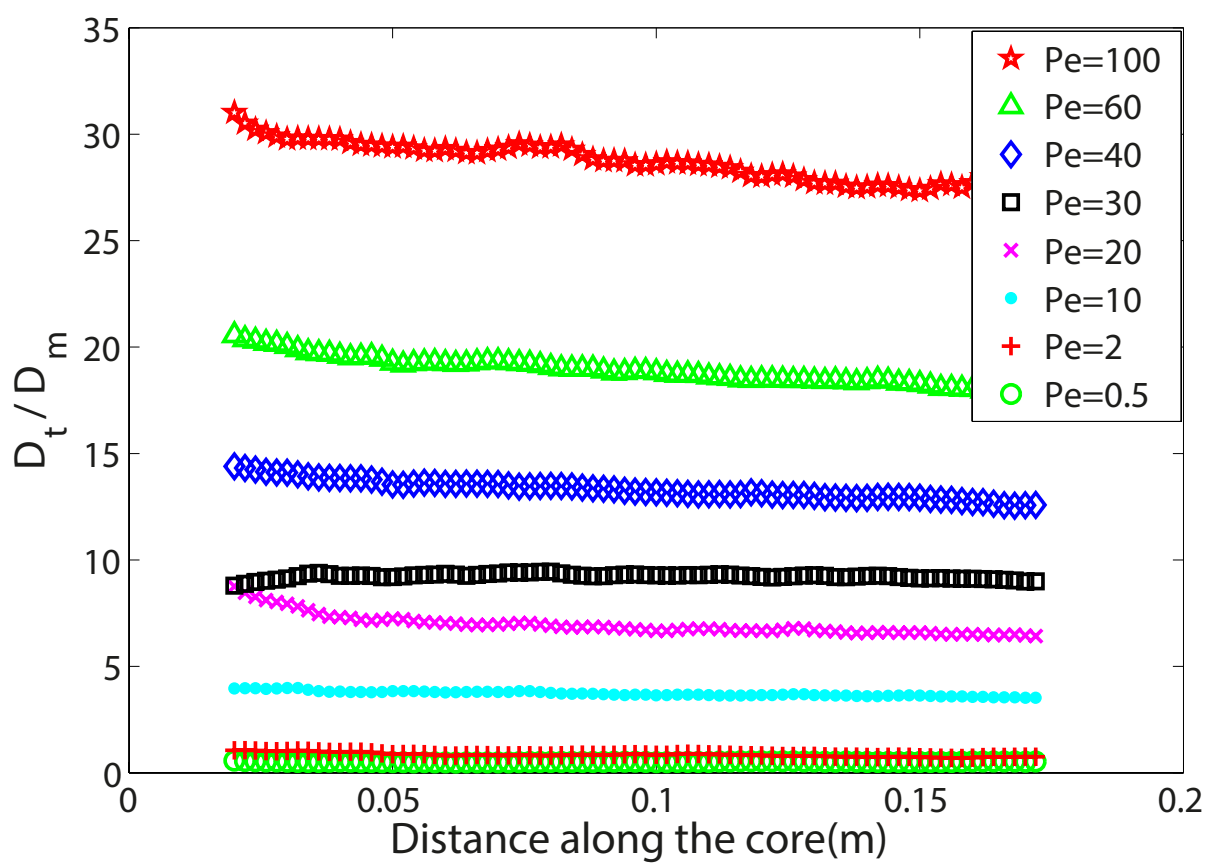

Figure 6: $D_{t} / D_{m}$ vs distance along the core. $D_{t}$ was obtained from fitting the analytical solution to the concentration profile.

aqueous solution into a Berea sandstone for $\mathrm{Pe}=0.5, \mathrm{Pe}=10$ and $\mathrm{Pe}=100$. It can be seen that the tracer plume was more focused for the high Pe value than for the low one where there was more spreading in the transverse direction. From these concentration profiles, the transverse dispersion coefficients were calculated using the methods described in section 4

\subsection{Transverse Dispersion Coefficients}

The value of $D_{t}$ did not stay constant throughout the core but decreased with distance along the core for all Pe (Fig. 6). The decrease became more pronounced with increasing Pe.

The core averaged transverse dispersion coefficients, obtained with the different methods for the different Pe can be found in Fig. 7 and Table 1 . For 


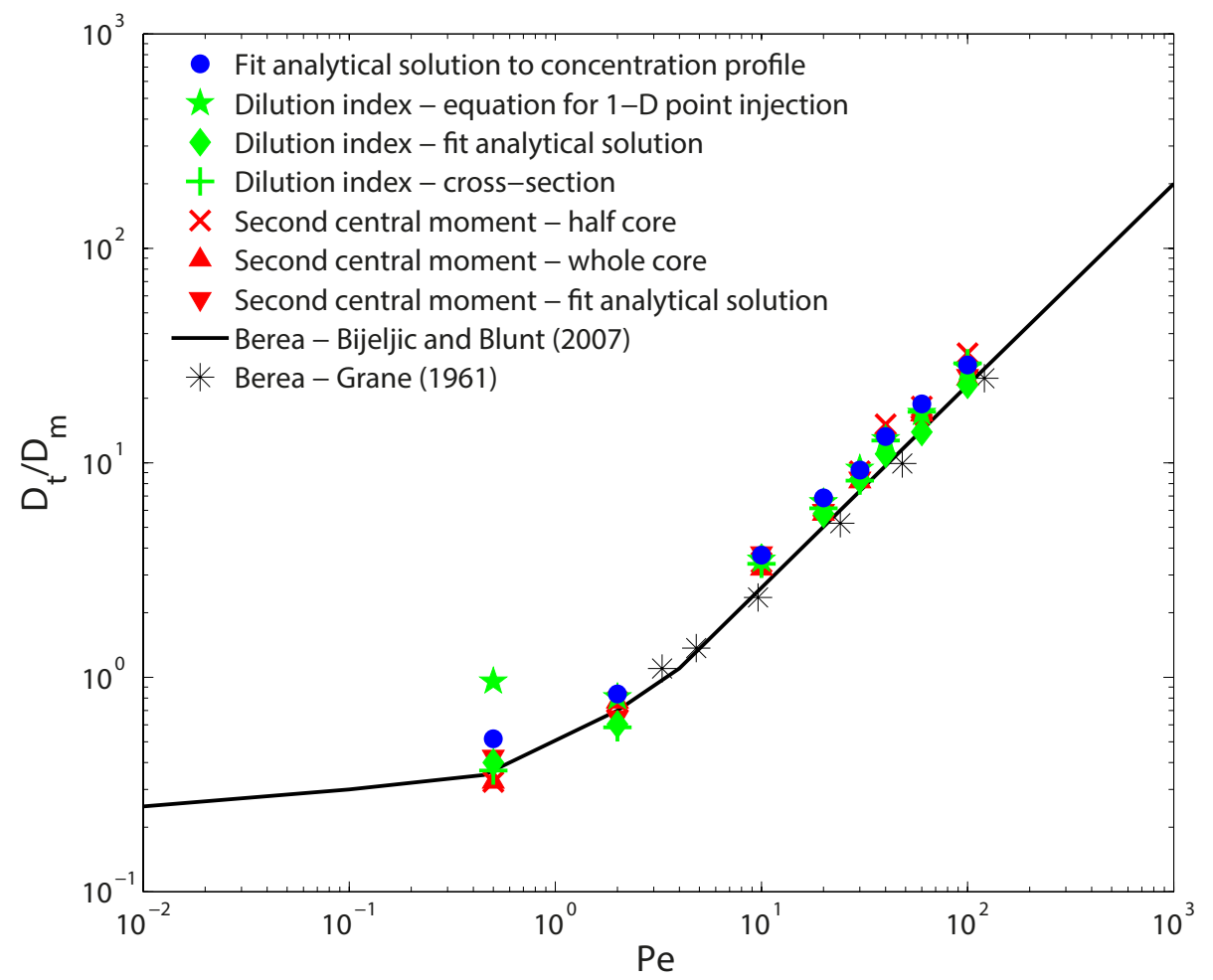

Figure 7: $D_{t} / D_{m}$ vs Pe. The colored symbols represent the results using the different methods to calculate $D_{t}$ for the Berea sandstone of this study. The black solid line shows the pore scale modeling result for a pore-size distribution of the Berea sandstone from Bijeljic and Blunt 3]. The black asterisks are the experimental results of Grane 24] for Berea sandstone.

the high Pe, the second central moment method using Eq. 18 was applied to calculate the $D_{t}$ using: (1) only the first half of the core and (2) the whole core. For the low Pe $(0.5$ and 2$)$ only the part of the core was used where the boundaries did not impact the steady state concentration profile. The values of $D_{t}$ calculated from the whole core are lower than when only half of the core was used. When the whole length of the core is considered, fitting the analytical solution to the concentration profile gives the highest values of $D_{t}$ and the lowest values are obtained with the method of fitting the 1-D dilution index length profile. The methods using the second central moment fall in between. 
Table 1: $D_{t} / D_{m}$ and $95 \%$ confidence bounds in parentheses.

\begin{tabular}{|c|c|c|c|c|c|c|}
\hline \multirow{2}{*}{$\begin{array}{c}\mathrm{Pe} \\
0.5\end{array}$} & \multicolumn{2}{|c|}{$\begin{array}{l}D_{t} / D_{m} \\
E-E q \cdot 23\end{array}$} & \multicolumn{2}{|c|}{$\begin{array}{c}D_{t} / D_{m} \\
E-\text { fit analytical }\end{array}$} & \multicolumn{2}{|c|}{$\begin{array}{c}D_{t} / D_{m} \\
E-\text { cross section }\end{array}$} \\
\hline & 0.956 & $(0.804-1.14)$ & 0.400 & $(0.375-4.25)$ & 0.367 & $(0.322-0.413)$ \\
\hline 2 & 0.808 & $(0.642-1.02)$ & 0.603 & $(0.574-0.631)$ & 0.584 & $(0.563-0.604)$ \\
\hline 10 & 3.53 & $(3.34-3.73)$ & 3.61 & $(3.58-3.64)$ & 3.38 & $(3.25-3.51)$ \\
\hline 20 & 6.55 & $(6.33-5.93)$ & 5.78 & $(5.68-5.89)$ & 6.13 & $(5.81-6.46)$ \\
\hline 30 & 9.38 & $(9.20-9.56)$ & 8.36 & $(8.24-8.47)$ & 8.26 & $(7.94-8.58)$ \\
\hline 40 & 12.9 & $(12.7-13.1)$ & 11.0 & $(10.9-11.2)$ & 12.7 & $(12.2-13.2)$ \\
\hline 60 & 17.1 & $(16.9-17.3)$ & 13.9 & $(13.7-14.2)$ & 17.3 & $(16.6-18.1)$ \\
\hline 100 & 27.6 & $(27.3-27.9)$ & 23.1 & $(22.3-23.9)$ & 29.1 & $(27.6-30.7)$ \\
\hline \multirow[t]{2}{*}{$\mathrm{Pe}$} & \multicolumn{2}{|r|}{$D_{t} / D_{m}$} & \multicolumn{2}{|r|}{$D_{t} / D_{m}$} & \multicolumn{2}{|r|}{$D_{t} / D_{m}$} \\
\hline & \multicolumn{2}{|c|}{$M_{2}-$ whole } & \multicolumn{2}{|r|}{$M_{2}-$ half } & \multicolumn{2}{|c|}{$M_{2}-$ fit analytical } \\
\hline 0.5 & 0.325 & $(0.314-0.337)$ & 0.325 & $(0.314-0.337)$ & 0.427 & $(0.404-0.450)$ \\
\hline 2 & 0.769 & $(0.720-0.818)$ & 0.769 & $(0.720-0.818)$ & 0.651 & $(0.629-0.673)$ \\
\hline 10 & 3.20 & $(3.17-3.22)$ & 3.43 & $(3.35-3.50)$ & 3.79 & $(3.76-3.83)$ \\
\hline 20 & 5.77 & $(5.73-5.82)$ & 6.22 & $(6.06-6.38)$ & 5.97 & $(5.89-6.05)$ \\
\hline 30 & 8.18 & $(8.11-8.24)$ & 9.12 & $(8.92-9.33)$ & 8.46 & $(8.35-8.57)$ \\
\hline 40 & 13.5 & $(13.3-13.7)$ & 15.1 & $(14.5-15.8)$ & 12.7 & $(12.5-13.0)$ \\
\hline 60 & 16.8 & $(16.5-17.1)$ & 18.3 & $(17.5-19.2)$ & 15.3 & $(15.1-15.6)$ \\
\hline 100 & 24.6 & $(24.2-25.1)$ & 32.4 & $(31.3-33.5)$ & 25.4 & $(24.3-26.5)$ \\
\hline \multirow[t]{2}{*}{$\mathrm{Pe}$} & \multicolumn{2}{|c|}{$D_{t} / D_{m}$} & & & & \\
\hline & \multicolumn{2}{|c|}{ fit conc. profile } & & & & \\
\hline 0.5 & 0.517 & $(0.463-0.571)$ & & & & \\
\hline 2 & 0.836 & $(0.772-0.900)$ & & & & \\
\hline 10 & 3.71 & $(3.62-3.80)$ & & & & \\
\hline 20 & 6.85 & $(6.69-7.01)$ & & & & \\
\hline 30 & 9.25 & $(9.05-9.45)$ & & & & \\
\hline 40 & 13.3 & $(12.9-13.6)$ & & & & \\
\hline 60 & 18.8 & $(18.3-19.3)$ & & & & \\
\hline 100 & 28.6 & $(27.6-29.7)$ & & & & \\
\hline
\end{tabular}




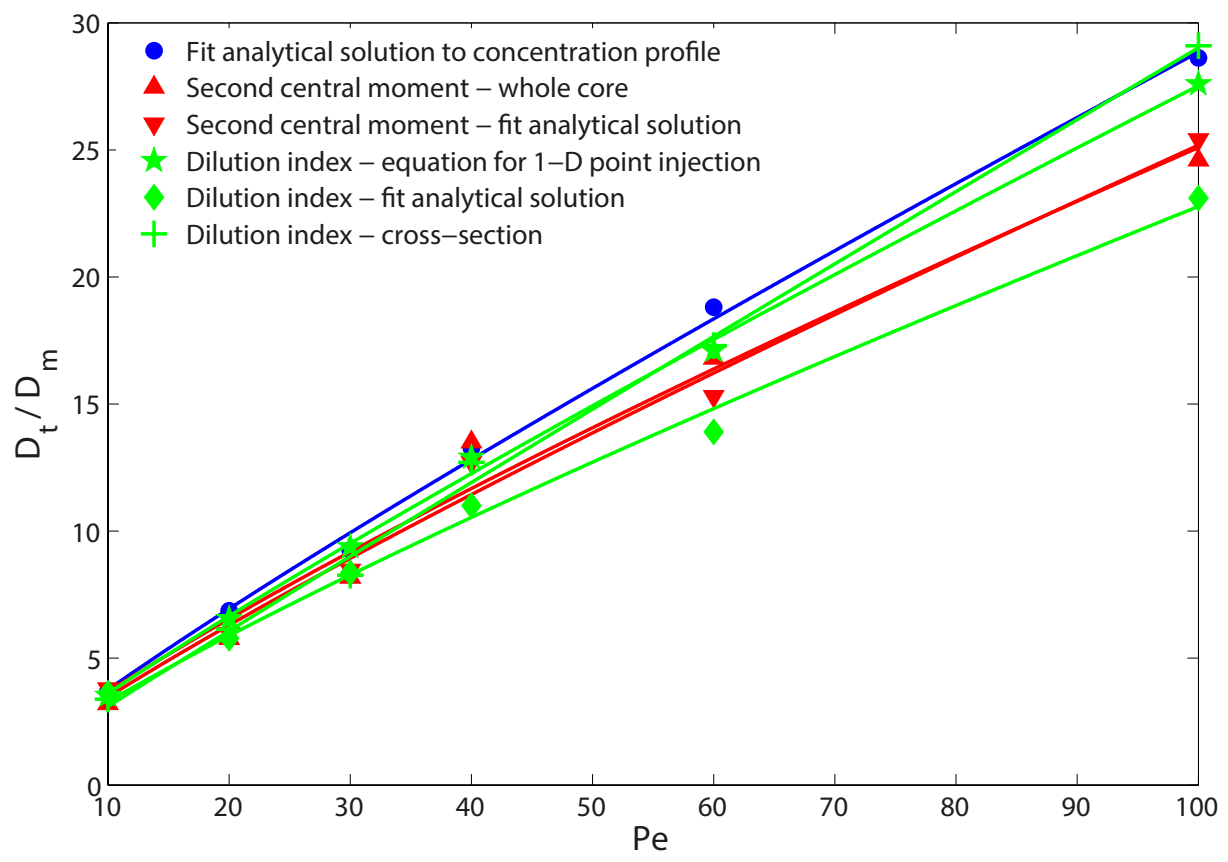

Figure 8: $D_{t} / D_{m}$ versus Pe in the power-law regime. Eq. 3 was fitted to this data. The values for $\beta$ and $\delta$ can be found in Table 2 .

The dependence of transverse dispersion on Pe for the power-law regime $(5<\mathrm{Pe}<300)$ was investigated by fitting Eq. 3 to the data. The fitted line can be seen in Fig. 8 and the values for $\beta$ and $\delta$ can be found in Table 2

355 The values for $\delta$ obtained with the different methods range from 0.84 to 0.97 indicating sub-linear behavior of transverse dispersion in the power-law regime.

\subsection{Transverse Dispersivities}

Fig. 9 shows the graph of $D_{t}$ versus velocity with straight lines fit to the data for the different methods where the line slopes provide the dispersivities. The dispersivities calculated this way can be found in Table 3 , together with the individually calculated dispersivities for each Pe studied. The dispersivities obtained from each individual Pe are generally of similar value within each method. Overall, the values of dispersivity of the methods of fitting the 1-D 
Table 2: $\quad \beta$ and $\delta$ values and $95 \%$ confidence bounds in parentheses from the fit to Eq. 3

\begin{tabular}{ccc}
\hline Method & $\beta$ & $\delta$ \\
\hline Conc. profile - fit analytical & $0.49(0.34-0.63)$ & $0.89(0.82-0.96)$ \\
$M_{2}-$ whole core & $0.53(0.11-0.96)$ & $0.84(0.65-1.0)$ \\
$M_{2}-$ fit analytical & $0.48(0.20-0.76)$ & $0.86(0.72-1.0)$ \\
$E-E q .23$ & $0.47(0.35-0.60)$ & $0.88(0.82-0.94)$ \\
$E-$ fit analytical & $0.47(0.27-0.67)$ & $0.84(0.74-0.94)$ \\
$E-$ cross section & $0.33(0.20-0.46)$ & $0.97(0.87-1.1)$ \\
\hline
\end{tabular}

Table 3: Core average dispersivity $\left(\alpha_{t}\right) \times 10^{-5} \mathrm{~m}$ calculated for each Pe and from the slope of Fig. 9.

\begin{tabular}{|c|c|c|c|c|c|c|}
\hline $\mathrm{Pe}$ & Conc. profile & $M_{2}-$ whole & $M_{2}-f i t$ & $E-E q \cdot 23$ & $E-f i t$ & $E-$ cross section \\
\hline 10 & 4.06 & 3.30 & 4.19 & 3.80 & 3.92 & 3.57 \\
\hline 20 & 4.39 & 3.58 & 3.73 & 4.16 & 3.59 & 3.85 \\
\hline 30 & 4.12 & 3.59 & 3.73 & 4.19 & 3.68 & 3.63 \\
\hline 40 & 4.60 & 4.69 & 4.39 & 4.47 & 3.75 & 4.39 \\
\hline 60 & 4.45 & 3.95 & 3.58 & 4.02 & 3.23 & 4.08 \\
\hline 100 & 4.14 & 3.54 & 3.66 & 3.99 & 3.32 & 4.22 \\
\hline Slope Fig. 9 & 4.17 & 3.60 & 3.58 & 3.97 & 3.20 & 4.30 \\
\hline
\end{tabular}

dilution index length profile, and fitting the analytical solution to the concentration profile stay most constant and act as a lower and upper limit, respectively.

\subsection{Dilution Index}

The dilution index was calculated for the experimental, analytical, numerical (CrunchFlow) results and from the equation defining the dilution index for instantaneous 1-D point injection (Eq. 23). The results can be seen in Fig. 10 The analytical, numerical and the instantaneous point injection results are identical for $\mathrm{Pe}=100$ but for $\mathrm{Pe}=10$ the result from the instantaneous point injection starts to deviate. This becomes more pronounced for $\mathrm{Pe}=2$ due to the radial 


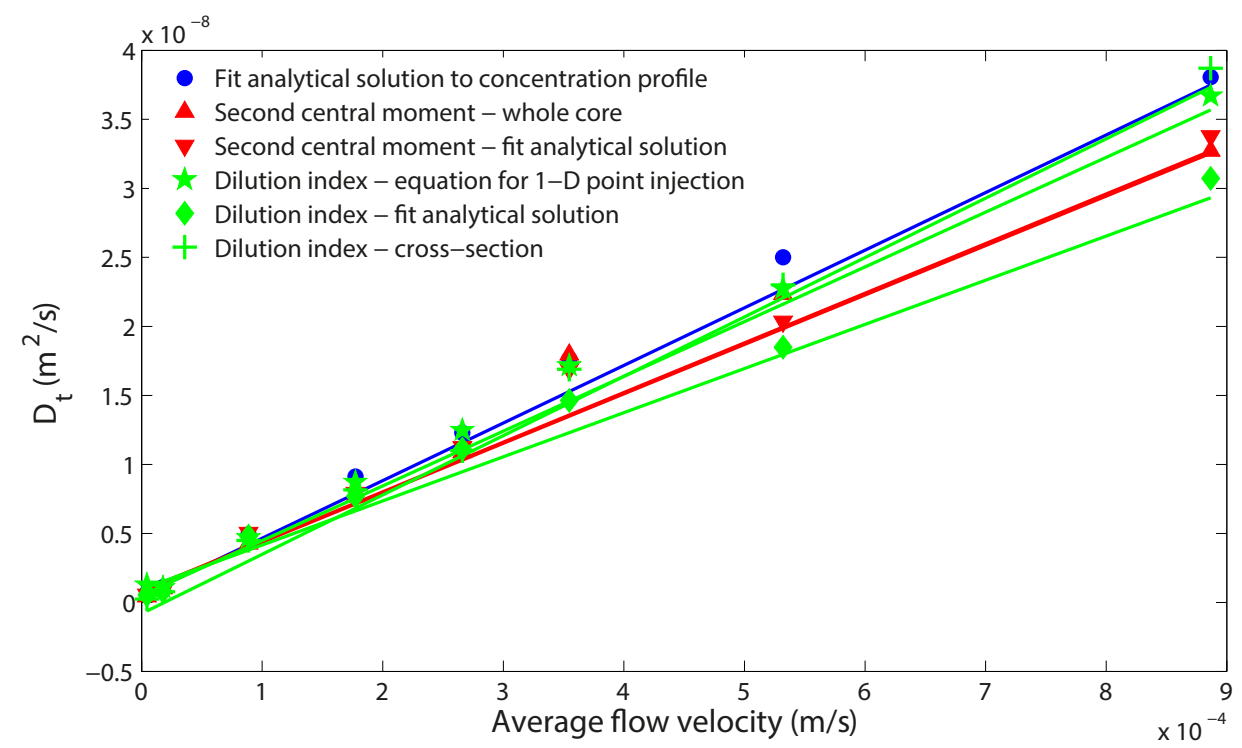

Figure 9: $D_{t}$ vs velocity using the different methods to calculate $D_{t}$. The dispersivity can be calculated from the slope of the best fit line.

boundary of the core. The experimentally obtained dilution index follows the same curve as the analytical and numerical dilution index initially. For $\mathrm{Pe}=2$ the index starts to deviate after $0.04 m$, for $\mathrm{Pe}=10$ after $0.09 \mathrm{~m}$, and for $\mathrm{Pe}=100$ after $0.12 m$. The slope of the plot of $\ln (\mathrm{E})$ versus $\ln (\mathrm{t})$ for the different Pe can be found in Table 4. The slope for a Gaussian plume would be $1 / 2$ - the slopes obtained from the experimental results are smaller but close to $1 / 2$ for all $\mathrm{Pe}$ studied.

To check whether the obtained values of $D_{t}$ for the different Pe are in agreement, the dilution index was calculated for the instantaneous point injection (Eq. 23) using the transverse dispersion coefficients obtained (i) from fitting the analytical solution to the concentration profile, (ii) from the dilution index method using the full cross-section, (iii) from fitting the second central moment length profile, and (iv) from fitting the dilution index length profile. The results can be seen in Fig. 11. The dilution index should decrease for higher Pe - this is only consistently the case for the transverse dispersion coefficients obtained 


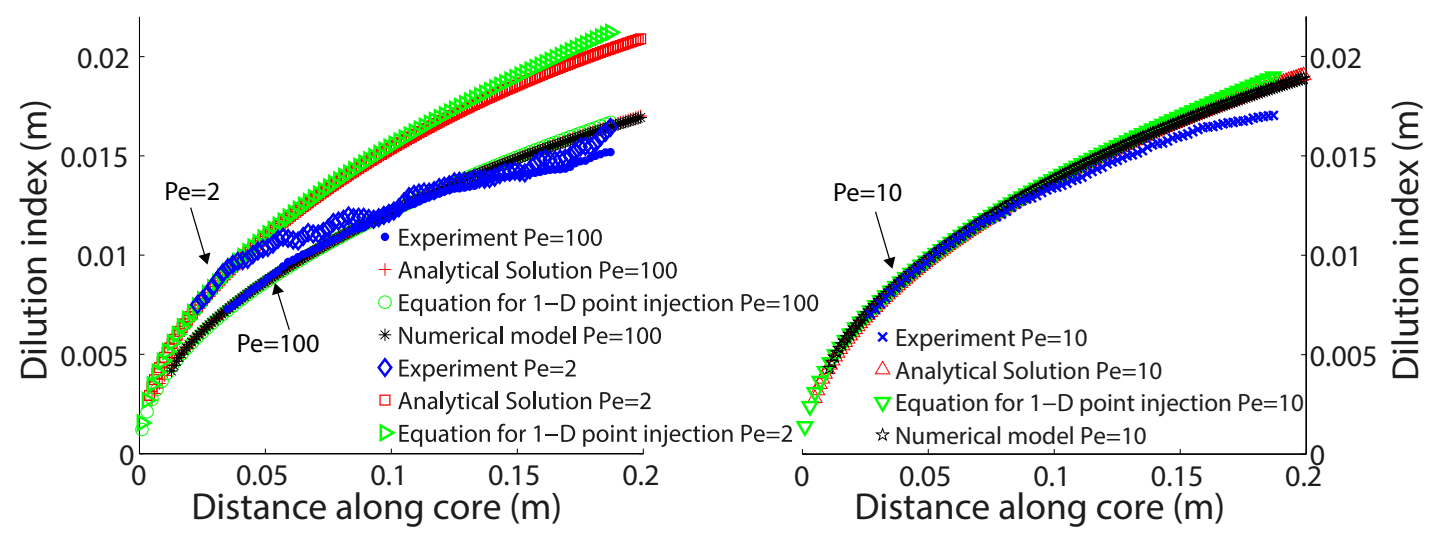

Figure 10: Dilution index vs distance for the experimental, analytical, numerical results and from the dilution index equation for point injection (Eq. 23) for $\mathrm{Pe}=2$ and 100 (left) and $\mathrm{Pe}=10$ (right).

from fitting the analytical solution to the concentration profile (with the exception of $\mathrm{Pe}=30$ ) and from fitting the dilution index length profile (with the exception of $\mathrm{Pe}=2$ ).

\subsection{Peak Concentrations}

Peak, or maximum concentrations within a transect weighted according to the area of the ring $\left(C_{\text {peak }}\right)$, were calculated for the experimental, analytical, numerical (CrunchFlow) results (Fig. 12). The peak concentration for $\mathrm{Pe}=100$ and 10 follow the same trend as the numerical result. For $\mathrm{Pe}=2$ the peak concentration stays much higher in the experiment than predicted by the model.

\subsection{Reactor Ratio}

The reactor ratio and the peak concentration ratio were obtained by dividing the experimentally obtained dilution index and peak concentration with the dilution index and peak concentration of the Gaussian plume of the numerical solution, respectively. The results can be seen in Fig. 13 and 14 and Table 4 

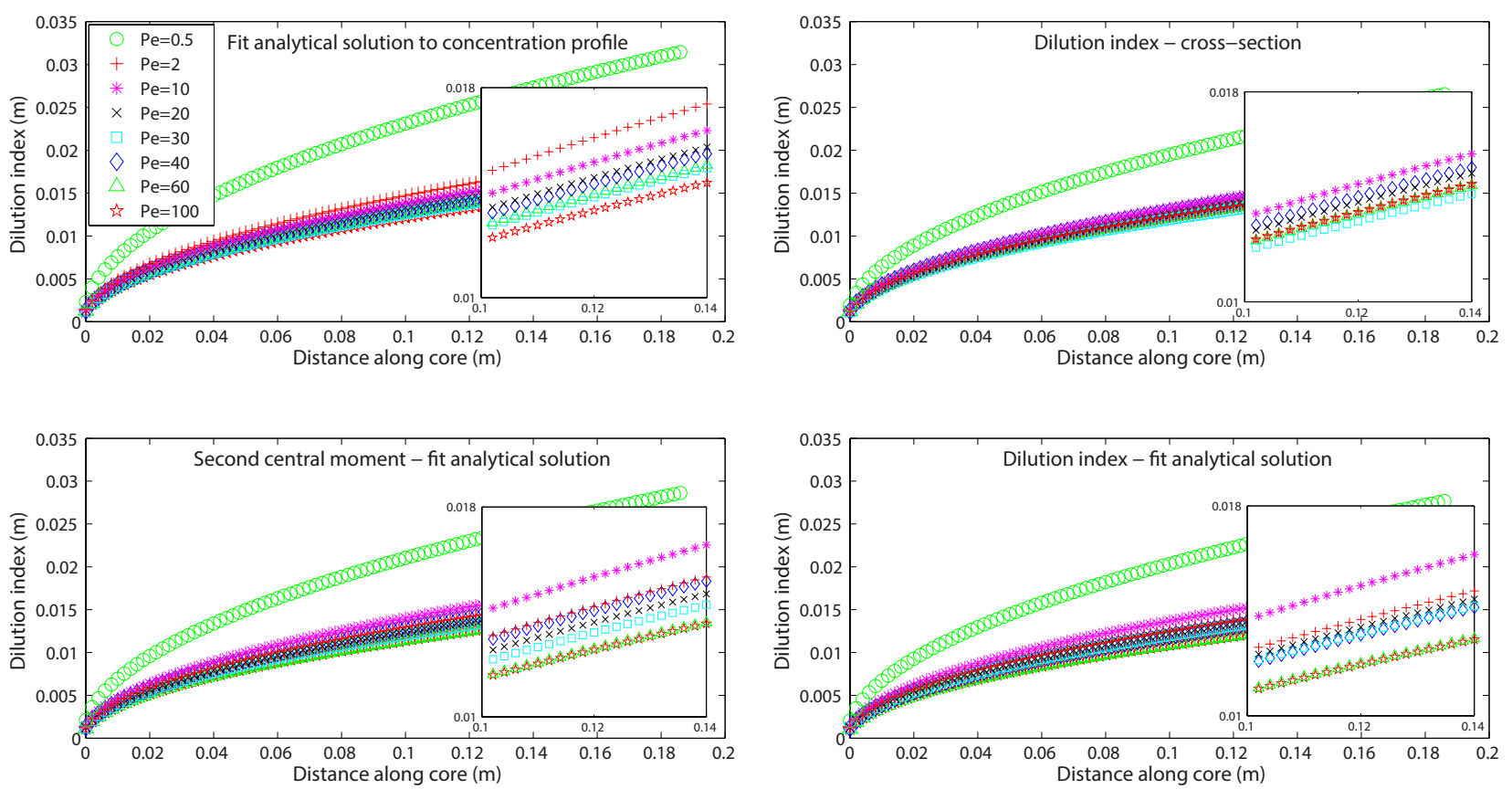

Figure 11: Dilution index calculated using Eq 23 with the $D_{t}$ obtained from fitting the analytical solution to the concentration profile (top left), from the dilution index - cross-section (top right), from fitting the second central moment length profile (bottom left), and from fitting the dilution index length profile (bottom right). 


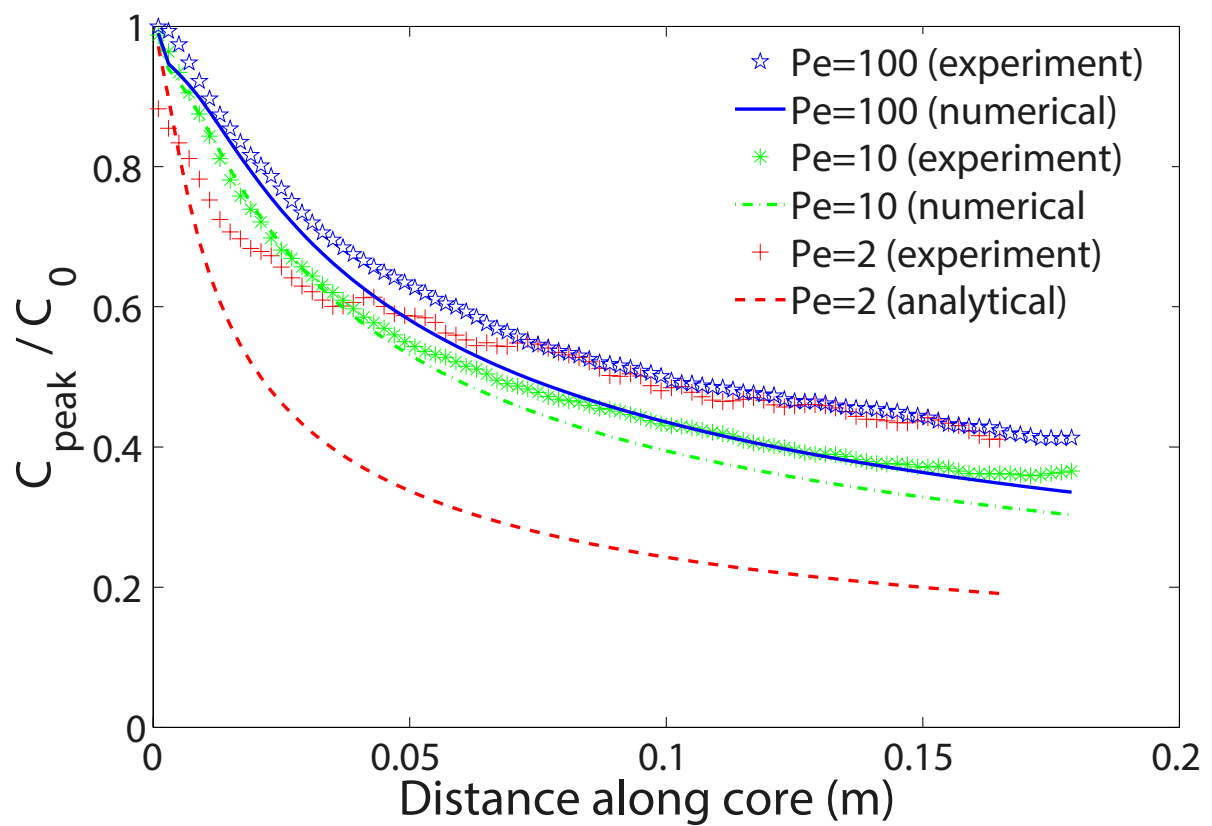

Figure 12: Normalized peak concentration versus distance for the experimental and numeri$\mathrm{cal} /$ analytical results for $\mathrm{Pe}=2,10$, and 100 . 


\begin{tabular}{cccc} 
Table 4 : Slope of $\ln (E)$ vs $\ln (t)$ graph, reactor ratio \& peak concentration ratio \\
\cline { 2 - 4 } Pe & slope & reactor ratio & peak concentration ratio \\
\hline 0.5 & 0.415 & 0.829 & 0.573 \\
2 & 0.495 & 0.810 & 0.930 \\
10 & 0.491 & 0.962 & 0.881 \\
20 & 0.482 & 0.945 & \\
30 & 0.487 & 0.951 & \\
40 & 0.481 & 0.945 & 0.888 \\
60 & 0.468 & 0.932 & \\
100 & 0.492 & 0.964 & \\
\hline
\end{tabular}
the core. There is a clear separation between the reactor ratios for high $\mathrm{Pe}$ in the power-law regime and the low $\mathrm{Pe}=2$ and 0.5 . Fig. 15 shows the $\ln (1 / E)$ and $\ln \left(C_{\text {peak }}\right)$ versus distance along the core. It can be seen that $\ln (1 / E)$ and $\ln \left(C_{\text {peak }}\right)$ follow the same trend.

\section{Discussion}

\subsection{Experimental Method}

The consistency between the observations of transverse dispersion observed in this work and those previously reported by Grane [24 and Bijeljic and Blunt [3] serves as a benchmark validating the use of this technique for observing solute transport and transverse dispersion. To that end, the core flood tests were rapid, and the simple experimental setup was relatively inexpensive as long as access to an X-ray CT scanner is available. The analytical solutions for the configuration allow for a simple design of the tests and also serve as a check for aberrations due to experimental artefacts. The similarity between the concentration distributions obtained with the two-dimensional ADE accounting for longitudinal dispersion and the solution neglecting longitudinal dispersion shows that longitudinal dispersion does not influence the steady-state concentration 


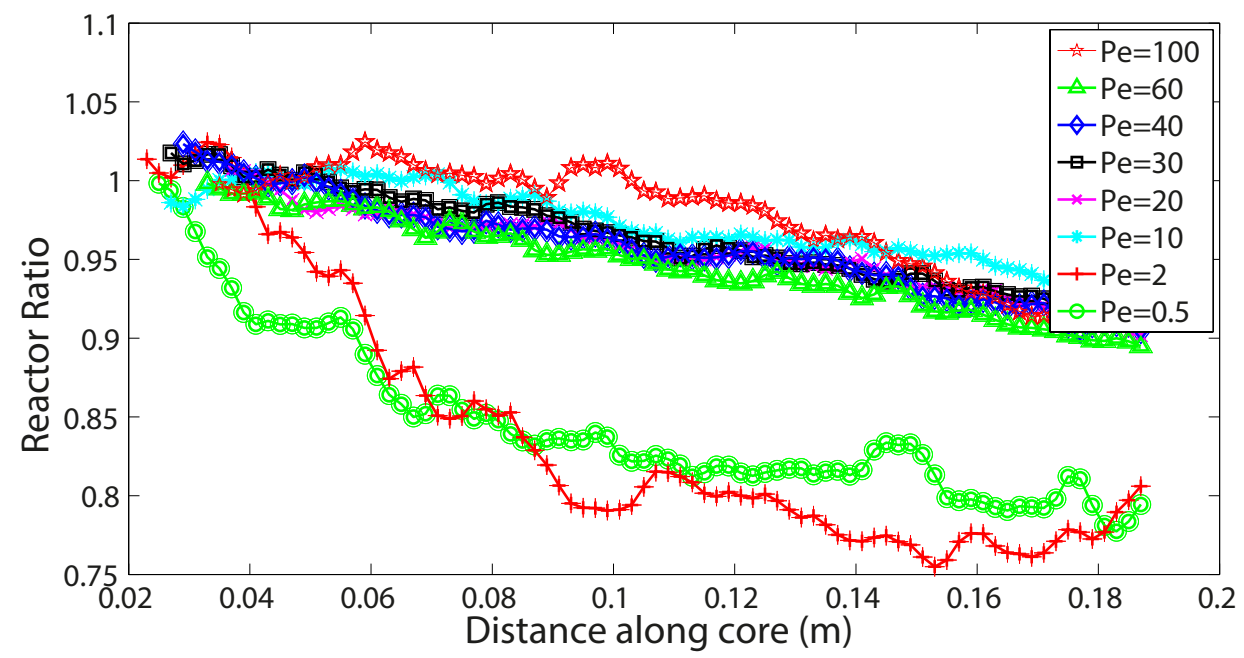

Figure 13: Reactor ratio (Eq 25 vs distance for the different Pe.

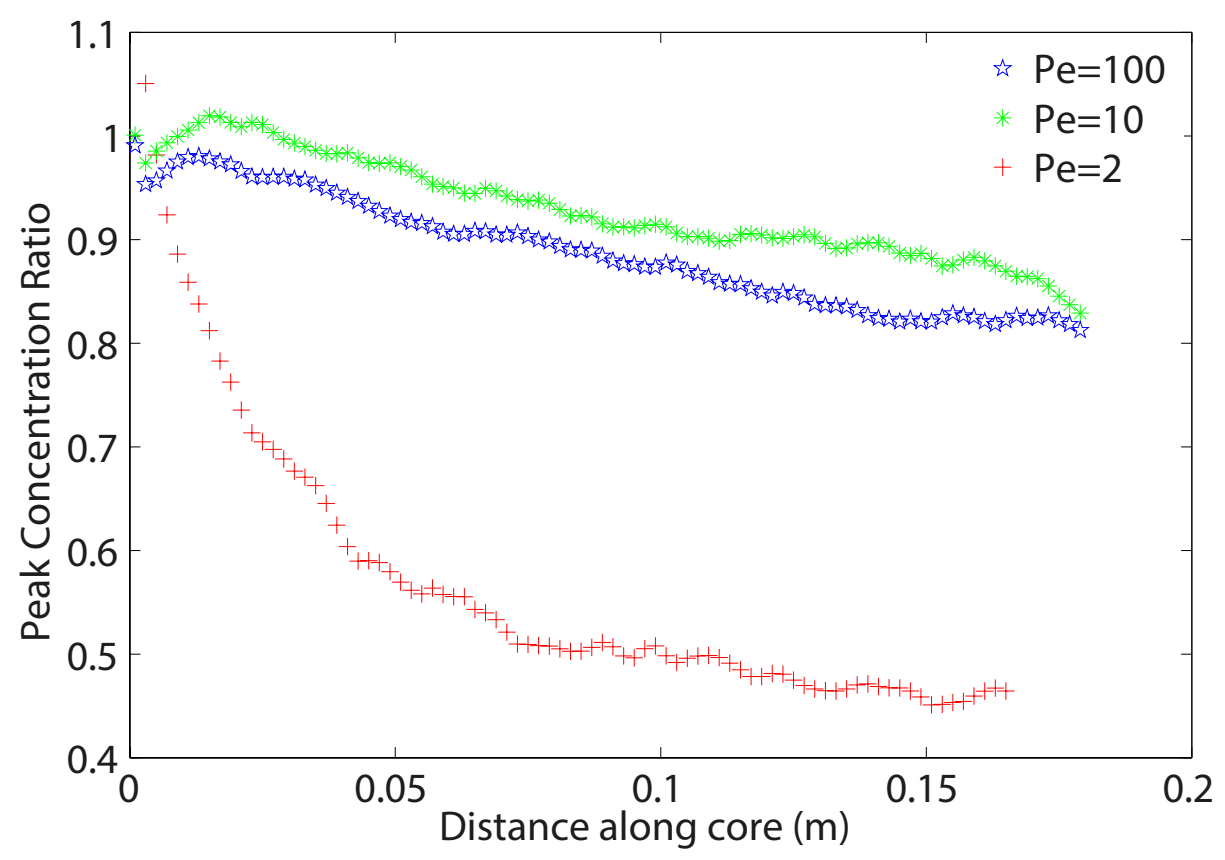

Figure 14: Peak concentration ratio vs distance for $\mathrm{Pe}=2,10$, and 100 . 


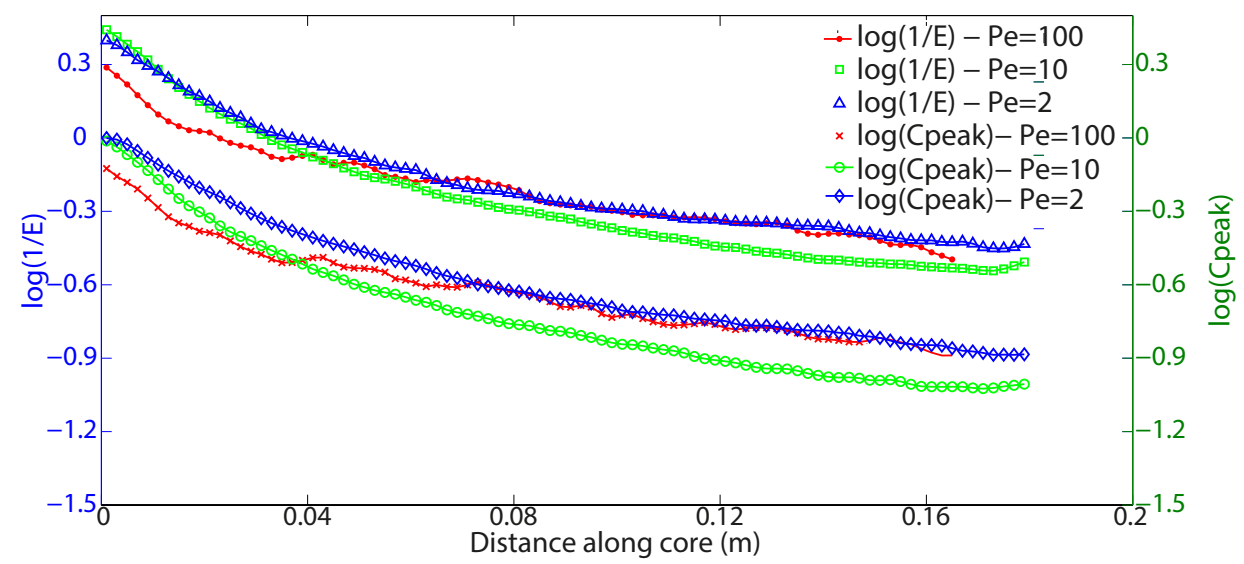

Figure 15: $\ln (1 / E)$ and $\ln \left(C_{\text {peak }}\right)$ vs distance.

profile. Similar observations have been made previously for different geometries 47.

The radial geometry of the core and the new annular inlet configuration used in the experiment (Fig 1) allow the transverse dispersion coefficients to be calculated using three different methods: (1) from fitting the analytical solution to the steady state concentration profile, (2) by analyzing the second-central moment, and (3) by analyzing the dilution index. This gives the opportunity to compare and estimate the precision of the different methods.

The analytical solution used from Eq. 17 is a solution for a homogeneous porous medium with the bounded geometry of our experiment and, therefore, the whole core can be used for the fitting. The second central moment method using Eq. 18 and the dilution index method using Eq. 23 work for both homogeneous and heterogeneous porous media but assume an unbounded domain which means that only the part of the core can be used where the radial boundaries do not impact the concentration profile.

Fig. 10 shows the dilution index obtained from the numerical simulations, the analytical solution and the equation for the dilution index for point injection in a homogeneous unbounded domain (Eq. 23) using the transverse dispersion coefficients obtained from the fit with the analytical solution. It can be seen 
that for $\mathrm{Pe}=10$ and 100 the boundaries do not impact the dilution index, i.e. the graphs fall on top of each other. For $\mathrm{Pe}=2$ it can be seen that the boundaries impact the dilution index and the graphs start to deviate. To calculate the transverse dispersion coefficients using the second central moment method and the dilution index method, the part of the core from the inlet to the point where they start to deviate was used.

To be able to use the whole core, the analytical solution was fitted to the second central moment length profile and the dilution index length profile.

\subsection{Transverse Dispersion and Dispersivity}

The transverse dispersion coefficient decreased with distance along the core (Fig. 6). This decrease was persistent and more pronounced for the higher Pe, which is an indication that this was not a boundary effect. Similar behavior was observed at the pore scale [3] and at the field scale in 2-D [19] and 3-D [18]. This behavior can be explained by the process of flow focusing where streamlines come together in zones of variable permeability. Due to this behavior the transverse dispersion coefficients were higher when calculated over half of the core (Table 1 and Fig. 7).

The core average transverse dispersion coefficients increased with $\mathrm{Pe}$ and showed a sub-linear dependence on Pe in the power-law regime with values of power-law coefficient $\delta$ ranging from 0.84 to 0.97 for the three methods (Table 2). This is in good agreement with the $\delta$ values obtained from pore scale modeling for Berea sandstone, $0.94[3]$ and 0.95+-0.07 [42] for the homogeneous Boise sandstone. Significantly lower experimentally obtained power-law coefficients have been reported in the literature for unconsolidated media, ranging from 0.5 for $\mathrm{Pe}>128$ for a sand pack [30, and 0.57 for $80<\mathrm{Pe}<1400$ for a sand pack 9 to 0.72 for $\mathrm{Pe}<6307$ for a bead and sand pack 34 . This sub-linear behavior has been attributed to incomplete mixing at the pore throats. Another possible explanation can be the more pronounced decrease in $D_{t}$ with distance for the higher Pe due to flow focusing.

The dispersivities calculated from the core average transverse dispersion co- 
efficients are generally of similar value within each method. Overall, the values of dispersivity of the methods of fitting the 1-D dilution index length profile, and fitting the analytical solution to the concentration profile stay most constant and act as a lower and upper limit, respectively. This shows that for a homogeneous rock like the Berea calculating the transverse dispersion coefficients for each of the radial transects is more precise than using the full cross-section because the width and location of the tracer can be adjusted during the fitting. Furthermore, the variation in dispersivities obtained from the fit in Fig. 9 for the different methods is relatively small, indicating that all methods can be used to obtain an estimate of the transverse dispersion coefficient.

\subsection{Dilution Index and Reactor Ratio}

Fig. 11 shows the dilution index calculated with the equation for instantaneous point injection (Eq. 23) by using the transverse dispersion coefficients obtained from the different methods. Physically the dilution index should decrease with Pe. This was observed for the transverse dispersion coefficients obtained from fitting the analytical solution to the concentration profile (except $\mathrm{Pe}=30)$ and from fitting the analytical solution to the dilution index length profile (except $\mathrm{Pe}=2$ ) but not for the $D_{t}$ obtained using the other methods. This indicates that fitting the analytical solution to the concentration profile and fitting the dilution index length profile are most precise in this case. However, for more heterogeneous rocks, fitting the analytical solution to the concentration profile might not work as the concentration distribution will not be close to a Gaussian distribution. Furthermore, rock heterogeneity can lead to extensive plume deformation affecting the symmetry of the system. When the system becomes asymmetrical the cross-sectional dilution index method will be the best option.

Fig. 13 and 14 show the reactor ratio and the peak concentration ratio, respectively, for the different Pe studied. It can be seen that both ratios follow the same trend for each of the Pe studied: the plume starts as a Gaussian plume and becomes slightly more irregular further along the core when more of the 
heterogeneities are sampled in the transverse direction. Both ratios decrease linearly with distance along the core and do not behave asymptotically. This can be explained by the fact that the transverse dispersion coefficient is not constant but decreases with distance (Fig. 6) while a constant $D_{t}$ is used in the numerical model. For the low $\mathrm{Pe}=0.5$ and 2 , the slope reaches an asymptotic value due to the radial boundary. The slope is initially much steeper for the low Pe because more of the core, and its heterogeneities, are sampled in the transverse direction resulting in a more irregular plume. The average reactor ratio for $\mathrm{Pe}=0.5$ and 2 is significantly lower, around 0.82 while the reactor ratio for the higher Pe is around 0.95 (Table 4 ).

The reactor ratios are high and follow the same trend indicated by the slope of the $\ln (\mathrm{E})$ versus $\ln (\mathrm{t})$ graph (close to a half, Table 4 ) and the inverse of the dilution index and the peak concentration following a similar trend (Fig. 15). 515 This all shows that the plume spreading stays very close to Gaussian behavior, which is expected for a homogeneous medium like the Berea sandstone.

In this test, sodium iodide was used because of the high attenuation at relatively low molar concentration. It is important to keep in mind that transverse dispersion is compound dependent [11, 12, 40]. In principle, the test is general to any compound of interest, as long as the impact on the local atomic density is sufficient to induce attenuation in the X-ray imagery. Thus, there will be a trade-off between molecular weight of a compound and its mole fraction in solution, so far as precision in the imaging is concerned. For Pe dependent experiments, the same compound can be used in order to quantify the contribution 525 of different physical mechanism.

\section{Conclusions}

We have developed a new core flood test for the characterization of transverse solute transport in three dimensions in natural-rock media. The test has been demonstrated to produce high precision observations of transverse dispersion and dilution over a broad range of Pe. Analytical solutions of the transport 
equation for this new core-flood test were derived that can be used in experimental design and analysis. Transverse dispersion coefficients were calculated by (1) fitting the analytical solution to the steady state concentration profile, by (2) analyzing the second-central moment, and by (3) analyzing the dilution index. All three methods give similar estimates of the transverse dispersion coefficients and can be used to estimate the transverse dispersion coefficient. However, the methods of fitting the analytical solution to the concentration profile, and the fit to the 1-D dilution index length profile seem to be most accurate and act as the upper and lower limit, respectively. For more heterogeneous rocks, where extensive deformations of solute plumes occur, the dilution index method using the full cross-section of the core might be the only method that can be applied. This is the subject of future work.

We have shown that at the core scale transverse dispersion coefficients decrease with distance due to flow focusing and that this effect was more pronounced for the higher Pe. This behavior has previously been observed for the core and field scale and can be a possible explanation for the sub-linear behavior of the increase of transverse dispersion coefficients with Pe observed in the power-law regime.

The plumes for the low Pe experiments were more irregular than the plumes

550 for the higher Pe because more pore-space heterogeneities were sampled in the transverse direction. Overall, the reactor ratios were high confirming the homogeneity of Berea sandstone.

\section{Appendix A. Derivation of Analytical Solutions to the Two Dimen- sional ADE}

The advection-dispersion equation (ADE) in cylindrical coordinates is given by:

$$
D_{l} \frac{\partial^{2} C}{\partial z^{2}}-v \frac{\partial C}{\partial z}+\frac{D_{t}}{r} \frac{\partial}{\partial r}\left(r \frac{\partial C}{\partial r}\right)=\frac{\partial C}{\partial t},
$$

where $C(z, r, t)$ is the solute concentration; $z$ is the distance along the core 
axis; $r$ is the radial distance; $t$ is the time; $v$ is the average water velocity (interstitial); $D_{l}$ and $D_{t}$ are the longitudinal and transverse dispersion coefficients, respectively. Initially, there is no solute in the system:

$$
C(z, r, t=0)=0 \quad 0 \leq z \leq \infty, \quad 0 \leq r \leq R,
$$

where $R$ is the radius of the core.

The solute is continuously injected into the middle annular region. By applying the law of mass conservation this can be described by

$$
v C(z=0, r, t)-D_{l} \frac{\partial C(z=0, r, t)}{\partial z}= \begin{cases}0 & 0 \leq r \leq \rho_{1} \\ v C_{0} & \rho_{1} \leq r \leq \rho_{2} \\ 0 & \rho_{2} \leq r \leq R\end{cases}
$$

where $C_{0}$ is the concentration of the injected solute; $\rho_{1}$ is the inner radius of the annular source; $\rho_{2}$ is the outer radius of the annular source. Furthermore, the system is assumed to be of semi-infinite length:

$$
C(z \rightarrow \infty, r, t)=0 .
$$

And impermeable conditions exist at $\mathrm{r}=\mathrm{R}$ :

$$
\frac{\partial C(z, r=R, t)}{\partial r}=0 .
$$

The analytical solutions, transient and steady state of the 2D ADE in cylindrical coordinates and the boundary conditions described above can be obtained following the same procedure as Chen et al. [10].

\section{Finite Hankel Transform}

First, the second kind of Finite Hankel transform is carried out on the ADE (Eq. A.1) with respect to $\mathrm{r}$ resulting in:

$$
D_{l} \frac{\partial^{2} C_{H}}{\partial z^{2}}-v \frac{\partial C_{H}}{\partial z}-D_{t} \lambda_{n}^{2} C_{H}=\frac{\partial C_{H}}{\partial t},
$$


where $\lambda_{n}$ is the Finite Hankel transform parameter which is determined by

$$
\frac{d J_{0}\left(\lambda_{n} R\right)}{d r}=0
$$

575 where $J_{0}()$ is the zero order Bessel function of the first kind. $C_{H}\left(\lambda_{n}, z, t\right)$ is the second kind of Finite Hankel transform for $C(r, z, t)$ as defined by the following conjugate equations:

$$
\begin{array}{r}
C_{H}\left(\lambda_{n}, z, t\right)=H[C(r, z, t)]=\int_{0}^{R} r C(r, z, t) J_{0}\left(\lambda_{n} r\right) d r \\
C(r, z, t)=\frac{2}{R^{2}} C_{H}\left(\lambda_{n}=0, z, t\right) \\
+\frac{2}{R^{2}} \sum_{n=1}^{\infty} C_{H}\left(\lambda_{n}, z, t\right) \frac{J_{0}\left(\lambda_{n} r\right)}{\left|J_{0}\left(\lambda_{n} R\right)\right|^{2}} .
\end{array}
$$

To obtain the transformed initial and boundary conditions the Finite Hankel transform is carried out over $\mathrm{r}$ :

580 for $\lambda_{n} \neq 0$ :

$$
\begin{array}{r}
\int_{\rho_{1}}^{\rho_{2}} r J_{0}\left(\lambda_{n} r\right) d r=\frac{1}{\lambda_{n}} \int_{\rho_{1}}^{\rho_{2}} \lambda_{n} r J_{0}\left(\lambda_{n} r\right) d r \\
\text { use } \frac{d}{d x}\left[x^{p} J_{p}(x)\right]=x^{p} J_{p-1}(x) \\
\frac{1}{\lambda_{n}^{2}}\left[\lambda_{n} r J_{1}\left(\lambda_{n} r\right)\right]_{\rho_{1}}^{\rho_{2}}=\frac{\rho_{2}}{\lambda_{n}} J_{1}\left(\lambda_{n} \rho_{2}\right)-\frac{\rho_{1}}{\lambda_{n}} J_{1}\left(\lambda_{n} \rho_{1}\right),
\end{array}
$$

for $\lambda_{n}=0$ :

$$
\begin{array}{r}
\int_{\rho_{1}}^{\rho_{2}} r J_{0}\left(\lambda_{n} r\right) d r=\int_{\rho_{1}}^{\rho_{2}} r J_{0}(0) d r \\
u s e \quad J_{0}(0)=1 \\
\int_{\rho_{1}}^{\rho_{2}} r d r=\left[\frac{1}{2} r^{2}\right]_{\rho_{1}}^{\rho_{2}}=\frac{\rho_{2}^{2}}{2}-\frac{\rho_{1}^{2}}{2} .
\end{array}
$$

The initial and boundary conditions after the Finite Hankel transform are:

$$
\begin{gathered}
C_{H}\left(\lambda_{n}, z, t=0\right)=0, \\
C_{H}\left(\lambda_{n}, z \rightarrow \infty, t\right)=0,
\end{gathered}
$$




$$
v C_{H}\left(\lambda_{n}, z=0, t\right)-D_{L} \frac{\partial C_{H}\left(\lambda_{n}, z=0, t\right)}{\partial z}=v C_{0} F\left(\lambda_{n}\right),
$$

585 where

$$
F\left(\lambda_{n}\right)= \begin{cases}\frac{\rho_{2}^{2}}{2}-\frac{\rho_{1}^{2}}{2} & \lambda_{n}=0 \\ \frac{\rho_{2}}{\lambda_{n}} J_{1}\left(\lambda_{n} \rho_{2}\right)-\frac{\rho_{1}}{\lambda_{n}} J_{1}\left(\lambda_{n} \rho_{1}\right) & \lambda_{n} \neq 0\end{cases}
$$

and $J_{1}()$ is the first order Bessel function of the first kind.

Laplace Trasnsform

Next, the Laplace transform is applied with respect to t:

$$
L\left[C_{H}\left(\lambda_{n}, z, t\right)\right]=C_{H L}\left(\lambda_{n}, z, s\right)=\int_{0}^{\infty} C_{H}\left(\lambda_{n}, z, t\right) e^{-s t} d t .
$$

$L\left(\frac{\partial C_{H}}{\partial t}\right)$ is, using $L\left(f^{\prime}(t)\right)=s F(s)-f(0)$, :

$$
\begin{array}{r}
L\left(C_{H}^{\prime}(t)\right)=s L\left(C_{H}\right)-C_{H}(0) \\
\text { where } \quad C_{H}(0)=0 \text { thus } \\
L\left(C_{H}^{\prime}(t)\right)=s L\left(C_{H}\right)=s C_{H L} .
\end{array}
$$

Substituting Eq. A.18 into Eq. A.6 results in:

$$
D_{l} \frac{\partial^{2} C_{H L}}{\partial z^{2}}-v \frac{\partial C_{H L}}{\partial z}-\left(D_{t} \lambda_{n}^{2}+s\right) C_{H L}=0 .
$$

After the Laplace transform Eq. A.13 and Eq. A.14 become:

$$
\begin{gathered}
C_{H L}\left(\lambda_{n}, z \rightarrow \infty, s\right)=0 \\
v C_{H L}\left(\lambda_{n}, z=0, s\right)-D_{l} \frac{\partial C_{H L}\left(\lambda_{n}, z=0, s\right)}{\partial z}= \\
\frac{v C_{0} F\left(\lambda_{n}\right)}{s} .
\end{gathered}
$$

( Laplace transform of a constant is $\frac{1}{s}$ ) 


\section{Particular Solution} tion:

$$
\begin{array}{r}
C_{H L}\left(\lambda_{n}, z, s\right)= \\
a_{1} \exp \left[\left(\frac{v+\sqrt{v^{2}+4 D_{l}\left(D_{t} \lambda_{n}^{2}+s\right)}}{2 D_{l}}\right) z\right] \\
+a_{2} \exp \left[\left(\frac{v-\sqrt{v^{2}+4 D_{l}\left(D_{t} \lambda_{n}^{2}+s\right)}}{2 D_{l}}\right) z\right] .
\end{array}
$$

The constants $a_{1}$ and $a_{2}$ can be found with the use of the boundary conditions (Eq. A.20 and Eq. A.21). The particular solution to this problem becomes:

$$
\begin{gathered}
C_{H L}\left(\lambda_{n}, z, s\right)=\frac{v C_{0} F\left(\lambda_{n}\right)}{\sqrt{D_{l}}} \exp \left(\frac{v z}{2 D_{l}}\right) \\
\frac{1}{s} \frac{\exp \left(-\frac{z}{\sqrt{D_{l}}} \sqrt{\frac{v^{2}}{4 D_{l}}+D_{t} \lambda_{n}^{2}+s}\right)}{\frac{v}{2 \sqrt{D_{l}}}+\sqrt{\frac{v^{2}}{4 D_{l}}+D_{t} \lambda_{n}^{2}+s}} .
\end{gathered}
$$

\section{Appendix A.1. Transient solution}

To obtain the transient solution in the original domain, $C(r, z, t)$, an inversion of the Laplace transform and the Finite Hankel transform need to be carried out.

\section{Inverse Laplace Transform}

First, the Laplace inverse transform is carried out on Eq. A.23 using the First Shift Theorem:

$$
L^{-1} F(s+a)(t)=e^{-a t} f(t) .
$$

For $\lambda_{n} \neq 0$, take $a=\frac{v^{2}}{4 D_{l}}+D_{t} \lambda_{n}^{2}$ and Eq. A.23 can be written as: 


$$
\begin{aligned}
& C_{H L}\left(\lambda_{n}, z, s\right)= \frac{v C_{0} F\left(\lambda_{n}\right)}{\sqrt{D_{l}}} \exp \left(\frac{v z}{2 D_{l}}\right) \\
& \frac{1}{s} \frac{\exp \left(-\frac{z}{\sqrt{D_{l}}} \sqrt{s+a}\right)}{\frac{v}{2 \sqrt{D_{l}}}+\sqrt{s+a}},
\end{aligned}
$$

610

where

$$
\frac{v C_{0} F\left(\lambda_{n}\right)}{\sqrt{D_{l}}} \exp \left(\frac{v z}{2 D_{l}}\right)=\text { constant }
$$

Therefore,

$$
\begin{array}{r}
C_{H}\left(\lambda_{n}, z, t\right)=\frac{v C_{0} F\left(\lambda_{n}\right)}{\sqrt{D_{l}}} \exp \left(\frac{v z}{2 D_{l}}\right) \\
L^{-1}\left[\frac{1}{s} \frac{\exp \left(-\frac{z}{\sqrt{D_{l}}} \sqrt{s+a}\right)}{\frac{v}{2 \sqrt{D_{l}}}+\sqrt{s+a}}\right] .
\end{array}
$$

Applying the First Shift Theorem A.24 results in:

$$
\begin{gathered}
C_{H}\left(\lambda_{n}, z, t\right)=\frac{v C_{0} F\left(\lambda_{n}\right)}{\sqrt{D_{l}}} \exp \left(\frac{v z}{2 D_{l}}\right) \\
\exp ^{-a t} L^{-1}\left[\frac{1}{s-a} \frac{\exp \left(-\frac{z}{\sqrt{D_{l}}} \sqrt{s}\right)}{\frac{v}{2 \sqrt{D_{l}}}+\sqrt{s}}\right] .
\end{gathered}
$$

Eq. A.27 can be solved with the following Laplace inverse formula:

$$
\begin{array}{r}
\mathrm{L}^{-1}\left[\frac{\exp (-\alpha \sqrt{s})}{\left(s-A^{2}\right)(B+\sqrt{s})}\right]= \\
\frac{1}{2(A+B)} \exp \left(A^{2} t-A \alpha\right) \operatorname{erfc}\left(\frac{\alpha}{2 \sqrt{t}}-A \sqrt{t}\right) \\
-\frac{1}{2(A-B)} \exp \left(A^{2} t+A \alpha\right) \operatorname{erfc}\left(\frac{\alpha}{2 \sqrt{t}}+A \sqrt{t}\right) \\
+\frac{B}{\left(A^{2}-B^{2}\right)} \exp \left(B^{2} t+B \alpha\right) \operatorname{erfc}\left(\frac{\alpha}{2 \sqrt{t}}+B \sqrt{t}\right) .
\end{array}
$$

Let $A=\left(\sqrt{\frac{v^{2}}{4 D_{l}}+D_{t} \lambda_{n}^{2}}\right), B=\left(\frac{v}{2 \sqrt{D_{l}}}\right)$ and $\alpha=\left(\frac{z}{\sqrt{D_{l}}}\right)$ then Eq. A.27 becomes:

$$
C_{H}\left(\lambda_{n}, z, t\right)=
$$




$$
\begin{array}{r}
C_{0} F\left(\lambda_{n}\right)\left\{\frac{v}{v+U} \exp \left[\left(\frac{v-U}{2 D_{l}}\right) z\right] \operatorname{erfc}\left(\frac{z-U t}{2 \sqrt{D_{l} t}}\right)\right. \\
+\frac{v}{v-U} \exp \left[\left(\frac{v+U}{2 D_{l}}\right) z\right] \operatorname{erfc}\left(\frac{z+U t}{2 \sqrt{D_{l} t}}\right) \\
\left.+\frac{v^{2}}{2 D_{l} D_{t} \lambda_{n}^{2}} \exp \left(\frac{v z}{D_{l}}-D_{t} \lambda_{n}^{2} t\right) \operatorname{erfc}\left(\frac{z+v t}{2 \sqrt{D_{l}}}\right)\right\},
\end{array}
$$

${ }_{615}$ where $U=v \sqrt{1+\frac{4 D_{l} D_{t} \lambda_{n}^{2}}{v^{2}}}$.

For $\lambda_{n}=0$ Eq. A.23 can be reduced to:

$$
\begin{aligned}
C_{H L}\left(\lambda_{n}=0, z, s\right)= & \frac{v C_{0} F\left(\lambda_{n}=0\right)}{\sqrt{D_{l}}} \exp \left(\frac{v z}{2 D_{l}}\right) \\
& \frac{1}{s} \frac{\exp \left(-\frac{z}{\sqrt{D_{l}}} \sqrt{\frac{v^{2}}{4 D_{l}}+s}\right)}{\frac{v}{2 \sqrt{D_{l}}}+\sqrt{\frac{v^{2}}{4 D_{l}}+s}} .
\end{aligned}
$$

Take $a=\frac{v^{2}}{4 D_{l}}$ :

$$
\begin{aligned}
C_{H L}\left(\lambda_{n}=0, z, s\right)=\frac{v C_{0} F\left(\lambda_{n}=0\right)}{\sqrt{D_{l}}} \exp \left(\frac{v z}{2 D_{l}}\right) \\
\frac{1}{s} \frac{\exp \left(-\frac{z}{\sqrt{D_{l}}} \sqrt{s+a}\right)}{\frac{v}{2 \sqrt{D_{l}}}+\sqrt{s+a}},
\end{aligned}
$$

where

$$
\frac{v C_{0} F\left(\lambda_{n}=0\right)}{\sqrt{D_{l}}} \exp \left(\frac{v z}{2 D_{l}}\right)=\text { constant. }
$$

620 Therefore,

$$
\begin{array}{r}
C_{H}\left(\lambda_{n}=0, z, t\right)=\frac{v C_{0} F\left(\lambda_{n}=0\right)}{\sqrt{D_{l}}} \exp \left(\frac{v z}{2 D_{l}}\right) \\
L^{-1}\left[\frac{1}{s} \frac{\exp \left(-\frac{z}{\sqrt{D_{l}}} \sqrt{s+a}\right)}{\frac{v}{2 \sqrt{D_{l}}}+\sqrt{s+a}}\right] .
\end{array}
$$


Applying the First Shift Theorem A.24 results in:

$$
\begin{array}{r}
C_{H}\left(\lambda_{n}=0, z, t\right)=\frac{v C_{0} F\left(\lambda_{n}=0\right)}{\sqrt{D_{l}}} \exp \left(\frac{v z}{2 D_{l}}\right) \\
\exp ^{-a t} L^{-1}\left[\frac{1}{s-a} \frac{\exp \left(-\frac{z}{\sqrt{D_{l}}} \sqrt{s}\right)}{\frac{v}{2 \sqrt{D_{l}}}+\sqrt{s}}\right] .
\end{array}
$$

Eq. A.33 can be solved with the following Laplace inverse formula:

$$
\begin{array}{r}
\mathrm{E}^{-1}\left[\frac{\exp (-\alpha \sqrt{s})}{\left(s-A^{2}\right)(A+\sqrt{s})}\right]= \\
\left(\frac{1}{\sqrt{\pi t}} \exp \left(\frac{\alpha^{2}}{4 t}\right)\right) t \\
+\frac{1}{4 A} \exp \left(A^{2} t-A \alpha\right) \operatorname{erfc}\left(\frac{\alpha}{2 \sqrt{t}}-A \sqrt{t}\right) \\
-\frac{1}{4 A}\left(1+2 A \alpha+4 A^{2} t\right) \exp \left(A^{2} t+A \alpha\right) \\
\operatorname{erfc}\left(\frac{\alpha}{2 \sqrt{t}}+A \sqrt{t}\right) .
\end{array}
$$

Let $A=\sqrt{\frac{v^{2}}{4 D_{l}}}=\frac{v}{2 \sqrt{D_{l}}}$ and $\alpha=\frac{z}{\sqrt{D_{l}}}$ then Eq. A.33 becomes:

$$
\begin{array}{r}
C_{H}\left(\lambda_{n}=0, z, t\right)=C_{0} F\left(\lambda_{n}=0\right) \\
\left\{\left(\sqrt{\frac{v^{2} t}{\pi D_{l}}} \exp \left(-\frac{(z-v t)^{2}}{4 D_{l} t}\right)+\frac{1}{2} \operatorname{erfc}\left[\frac{z-v t}{2 \sqrt{D_{l} t}}\right]\right.\right. \\
-\frac{1}{2}\left(1+\frac{v z}{D_{l}}+\frac{v^{2} t}{D_{l}}\right) \exp \left[\frac{v z}{D_{l}}\right] \operatorname{erfc}\left[\left(\frac{z+v t}{2 \sqrt{D_{l} t}}\right]\right\}
\end{array}
$$

Inverse Finite Hankel Transform

Next, the inverse Finite Hankel transform needs to be carried out using Eq.

A.9. Substituting Eq. A.29 and Eq. A.35 into Eq. A.9 results in:

$$
\begin{array}{r}
C(r, z, t)=\frac{C_{0}}{R^{2}}\left(\rho_{2}^{2}-\rho_{1}^{2}\right) \\
\left\{\left(\sqrt{\frac{v^{2} t}{\pi D_{l}}} \exp \left(-\frac{(z-v t)^{2}}{4 D_{l} t}\right)+\frac{1}{2} \operatorname{erfc}\left[\frac{z-v t}{2 \sqrt{D_{l} t}}\right]\right.\right. \\
-\frac{1}{2}\left(1+\frac{v z}{D_{L}}+\frac{v^{2} t}{D_{l}}\right) \exp \left[\frac{v z}{D_{l}}\right] \operatorname{erfc}\left[\left(\frac{z+v t}{2 \sqrt{D_{l} t}}\right]\right\}
\end{array}
$$




$$
\begin{array}{r}
+\frac{2}{R^{2}} \sum_{n=1}^{\infty} C_{0}\left(\frac{\rho_{2}}{\lambda_{n}} J_{1}\left(\lambda_{n} \rho_{2}\right)-\frac{\rho_{1}}{\lambda_{n}} J_{1}\left(\lambda_{n} \rho_{1}\right)\right) \\
\left\{\frac{v}{v+U} \exp \left[\left(\frac{v-U}{2 D_{l}}\right) z\right] \operatorname{erfc}\left(\frac{z-U t}{2 \sqrt{D_{l} t}}\right)\right. \\
+\frac{v}{v-U} \exp \left[\left(\frac{v+U}{2 D_{l}}\right) z\right] \operatorname{erfc}\left(\frac{z+U t}{2 \sqrt{D_{l}} t}\right) \\
\left.+\frac{v^{2}}{2 D_{l} D_{t} \lambda_{n}^{2}} \exp \left(\frac{v z}{D_{l}}-D_{t} \lambda_{n}^{2} t\right) \operatorname{erfc}\left(\frac{z+v t}{2 \sqrt{D_{l} t}}\right)\right\} \\
\frac{J_{0}\left(\lambda_{n} r\right)}{\left|J_{0}\left(\lambda_{n} R\right)\right|^{2}} .
\end{array}
$$

Eq. A.36 is the transient solution of the 2-D ADE for the boundary conditions described previously.

\section{Appendix A.2. Steady State Solution}

The steady state solution of the 2-D ADE for the boundary conditions described previously can be found by applying the Tauberian Theorem on Eq. A.23.

for $\lambda_{n} \neq 0$ :

$$
\begin{array}{r}
C_{H}\left(\lambda_{n}, z\right)=\frac{v C_{0}\left(\frac{\rho_{2}}{\lambda_{n}} J_{1}\left(\lambda_{n} \rho_{2}\right)-\frac{\rho_{1}}{\lambda_{n}} J_{1}\left(\lambda_{n} \rho_{1}\right)\right)}{\sqrt{D_{L}}} \\
\exp \left(\frac{v z}{2 D_{l}}\right) \frac{\exp \left(-\frac{z}{\sqrt{D_{l}}} \sqrt{\frac{v^{2}}{4 D_{l}}+D_{t} \lambda_{n}^{2}}\right)}{\frac{v}{2 \sqrt{D_{l}}}+\sqrt{\frac{v^{2}}{4 D_{l}}+D_{t} \lambda_{n}^{2}}},
\end{array}
$$

for $\lambda_{n}=0$ :

$$
\begin{array}{r}
C_{H}\left(\lambda_{n}=0, z\right)=\frac{v C_{0}\left(\rho_{2}^{2}-\rho_{1}^{2}\right)}{\sqrt{D_{l}}} \exp \left(\frac{v z}{2 D_{l}}\right) \\
\frac{\exp \left(-\frac{z}{\sqrt{D_{l}}} \sqrt{\frac{v^{2}}{4 D_{l}}}\right)}{\frac{v}{2 \sqrt{D_{l}}}+\sqrt{\frac{v^{2}}{4 D_{l}}}} .
\end{array}
$$

Next, the inverse Finite Hankel transform needs to be carried out using Eq. A.9 Substituting Eq. A.37 and Eq. A.38 into Eq. A.9 results in: 


$$
\begin{array}{r}
C(r, z, t)= \\
\frac{2}{R^{2}} \frac{v C_{0}\left(\rho_{2}^{2}-\rho_{1}^{2}\right)}{\sqrt{D_{l}}} \exp \left(\frac{v z}{2 D_{l}}\right) \frac{\exp \left(-\frac{z}{\sqrt{D_{l}}} \sqrt{\frac{v^{2}}{4 D_{l}}}\right)}{\frac{v}{2 \sqrt{D_{l}}}+\sqrt{\frac{v^{2}}{4 D_{l}}}} \\
+\frac{2}{R^{2}} \sum_{n=1}^{\infty} \frac{v C_{0}\left(\frac{\rho_{2}}{\lambda_{n}} J_{1}\left(\lambda_{n} \rho_{2}\right)-\frac{\rho_{1}}{\lambda_{n}} J_{1}\left(\lambda_{n} \rho_{1}\right)\right)}{\sqrt{D_{L}}} \\
\exp \left(\frac{v z}{2 D_{l}}\right) \frac{\exp \left(-\frac{z}{\sqrt{D_{l}}} \sqrt{\frac{v^{2}}{4 D_{l}}+D_{t} \lambda_{n}^{2}}\right)}{\frac{v}{2 \sqrt{D_{l}}}+\sqrt{\frac{v^{2}}{4 D_{l}}+D_{t} \lambda_{n}^{2}}} \frac{J_{0}\left(\lambda_{n} r\right)}{\left|J_{0}\left(\lambda_{n} R\right)\right|^{2}} .
\end{array}
$$

Eq. A.39 is the steady state solution of the 2-D ADE for the boundary conditions described previously.

Appendix B. Derivation of Analytical Solution to the 2-D ADE Ignoring Longitudinal Dispersion

When longitudinal dispersion is ignored, the ADE in cylindrical coordinates A.1) at steady state can be written as:

$$
\frac{D_{t}}{r} \frac{\partial}{\partial r}\left(r \frac{\partial C}{\partial r}\right)=v \frac{\partial C}{\partial z} .
$$

At the inlet of the core, the concentration in the middle annular region is $C_{0}$ and zero everywhere else:

$$
C(r, z=0)= \begin{cases}0 & 0 \leq r \leq \rho_{1} \\ C_{0} & \rho_{1} \leq r \leq \rho_{2} \\ 0 & \rho_{2} \leq r \leq R\end{cases}
$$

645

and no-flux conditions exist at $\mathrm{r}=\mathrm{R}$ :

$$
\frac{\partial C(r=R, z)}{\partial r}=0
$$


The steady state solution of the 2-D ADE ignoring $D_{l}$ in cylindrical coordinates and the boundary conditions described above can be obtained by first carrying out the second kind of Finite Hankel transform on Eq B.1 with respect to $r$ resulting in:

$$
-D_{t} \lambda_{n}^{2} C_{H}=v \frac{\partial C_{H}}{\partial z},
$$

where $\lambda_{n}$ is the Finite Hankel transform parameter which is determined by

$$
\frac{d J_{0}\left(\lambda_{n} R\right)}{d r}=0,
$$

where $J_{0}()$ is the zero order Bessel function of the first kind. $C_{H}\left(\lambda_{n}, z\right)$ is the second kind of Finite Hankel transform for $C(r, z)$ as defined by the following conjugate equations:

$$
\begin{array}{r}
C_{H}\left(\lambda_{n}, z\right)=H[C(r, z)]=\int_{0}^{R} r C(r, z) J_{0}\left(\lambda_{n} r\right) d r, \\
C(r, z)=\frac{2}{R^{2}} C_{H}\left(\lambda_{n}=0, z\right) \\
+\frac{2}{R^{2}} \sum_{n=1}^{\infty} C_{H}\left(\lambda_{n}, z\right) \frac{J_{0}\left(\lambda_{n} r\right)}{\left|J_{0}\left(\lambda_{n} R\right)\right|^{2}} .
\end{array}
$$

To obtain the transformed initial and boundary conditions the Finite Hankel transform is carried out over $\mathrm{r}$ :

for $\lambda_{n} \neq 0$ :

$$
\begin{array}{r}
\int_{\rho_{1}}^{\rho_{2}} r J_{0}\left(\lambda_{n} r\right) d r=\frac{1}{\lambda_{n}} \int_{\rho_{1}}^{\rho_{2}} \lambda_{n} r J_{0}\left(\lambda_{n} r\right) d r \\
\text { use } \frac{d}{d x}\left[x^{p} J_{p}(x)\right]=x^{p} J_{p-1}(x) \\
\frac{1}{\lambda_{n}^{2}}\left[\lambda_{n} r J_{1}\left(\lambda_{n} r\right)\right]_{\rho_{1}}^{\rho_{2}}=\frac{\rho_{2}}{\lambda_{n}} J_{1}\left(\lambda_{n} \rho_{2}\right)-\frac{\rho_{1}}{\lambda_{n}} J_{1}\left(\lambda_{n} \rho_{1}\right),
\end{array}
$$

for $\lambda_{n}=0$ :

$$
\int_{\rho_{1}}^{\rho_{2}} r J_{0}\left(\lambda_{n} r\right) d r=\int_{\rho_{1}}^{\rho_{2}} r J_{0}(0) d r
$$




$$
\begin{array}{r}
\text { use } \quad J_{0}(0)=1 \\
\int_{\rho_{1}}^{\rho_{2}} r d r=\left[\frac{1}{2} r^{2}\right]_{\rho_{1}}^{\rho_{2}}=\frac{\rho_{2}^{2}}{2}-\frac{\rho_{1}^{2}}{2} .
\end{array}
$$

The initial conditions after the Finite Hankel transform are:

$$
C\left(\lambda_{n}, z=0\right)=C_{0} F\left(\lambda_{n}\right)
$$

660

where

$$
F\left(\lambda_{n}\right)= \begin{cases}\frac{\rho_{2}^{2}}{2}-\frac{\rho_{1}^{2}}{2} & \lambda_{n}=0 \\ \frac{\rho_{2}}{\lambda_{n}} J_{1}\left(\lambda_{n} \rho_{2}\right)-\frac{\rho_{1}}{\lambda_{n}} J_{1}\left(\lambda_{n} \rho_{1}\right) & \lambda_{n} \neq 0\end{cases}
$$

and $J_{1}()$ is the first order Bessel function of the first kind.

Eq. B.4 is a homogeneous ordinary equation with the following general solution:

$$
C_{H}\left(\lambda_{n}, z\right)=c_{1} \exp \left(-D_{t} \lambda_{n}^{2} \frac{z}{v}\right)
$$

The constant $c_{1}$ can be found with the use of the initial conditions (Eq.

665 B.10. The particular solution to this problem becomes:

$$
C_{H}\left(\lambda_{n}, z\right)=C_{0} F\left(\lambda_{n}\right) \exp \left(-D_{t} \lambda_{n}^{2} \frac{z}{v}\right)
$$

To go back to the solution in the original domain, $C(r, z)$, an inversion of the Finite Hankel transform needs to be carried out using Eq. B.7. This results in:

$$
\begin{array}{r}
C(r, z)=\frac{C_{0}}{R^{2}}\left(\rho_{2}^{2}-\rho_{1}^{2}\right) \\
+\frac{2}{R^{2}} \sum_{n=1}^{\infty} C_{0}\left(\frac{\rho_{2}}{\lambda_{n}} J_{1}\left(\lambda_{n} \rho_{2}\right)-\frac{\rho_{1}}{\lambda_{n}} J_{1}\left(\lambda_{n} \rho_{1}\right)\right) \exp \left(-D_{t} \lambda_{n}^{2} \frac{z}{v}\right) \frac{J_{0}\left(\lambda_{n} r\right)}{\left|J_{0}\left(\lambda_{n} R\right)\right|^{2}} .(\mathrm{B}
\end{array}
$$

Eq. B.14 is the analytical solution of the 2-D ADE at steady state when longitudinal dispersion is ignored for the boundary conditions described above.

\section{Acknowledgments}

This work was carried out as part of the Qatar Carbonates and Carbon Storage Research Centre (QCCSRC). The authors gratefully acknowledge the 
funding of QCCSRC provided jointly by Qatar Petroleum, Shell, and the Qatar

Science \& Technology Park and for supporting the present project and the

permission to publish this research.

The authors would like to thank the editor, two anonymous reviewers and

Olaf Cirpka for their very helpful comments and suggestions.

\section{References}

[1] Anderson, M. P., and J. A. Cherry (1979), Using models to simulate the movement of contaminants through groundwater flow systems, Critical Reviews in Environmental Science and Technology, 9(2), 97-156.

[2] Benekos, I. D., O. A. Cirpka, and P. K. Kitanidis (2006), Experimental determination of transverse dispersivity in a helix and a cochlea, Water Resources Research, 42(7), doi:10.1029/2005WR004712, w07406.

[3] Bijeljic, B., and M. J. Blunt (2007), Pore-scale modeling of transverse dispersion in porous media, Water Resources Research, 43(12), doi:10.1029/ 2006WR005700.

[4] Bijeljic, B., P. Mostaghimi, and M. Blunt (2011), Signature of non-fickian solute transport in complex heterogeneous porous media, Physical Review Letters, 107, doi:10.1103/PhysRevLett.107.204502.

[5] Bijeljic, B., A. Raeini, P. Mostaghimi, and M. J. Blunt (2013), Predictions of non-fickian solute transport in different classes of porous media using direct simulation on pore-scale images, Physical Review E, 87(1), 013,011.

[6] Blackwell, R. J. (1962), Laboratory studies of microscopic dispersion phenomena, Society of Petroleum Engineers Journal, 2, 1-8, doi:10.2118/ $1483-\mathrm{G}$.

[7] Bolster, D., D. A. Benson, M. M. Meerschaert, and B. Baeumer (2013), Mixing-driven equilibrium reactions in multidimensional fractional advectiondispersion systems, Physica A: Statistical Mechanics and its Applica- 
tions, 392(10), 2513 - 2525, doi:http://dx.doi.org/10.1016/j.physa.2012. 12.040 .

[8] Borgne, T. L., T. R. Ginn, and M. Dentz (2014), Impact of fluid deformation on mixing-induced chemical reactions in heterogeneous flows, Geophysical Research Letters, 41(22), 7898-7906, doi:10.1002/2014GL062038.

[9] Carvalho de, J. R. F. G., and J. M. P. Q. Delgado (2000), Lateral dispersion in liquid flow through packed beds at pem i 1,400, AIChE Journal, 46(5), 1089-1095, doi:10.1002/aic.690460520.

[10] Chen, J.-S., Y.-H. Liu, C.-P. Liang, C.-W. Liu, and C.-W. Lin (2011), Exact analytical solutions for two-dimensional advectiondispersion equation in cylindrical coordinates subject to third-type inlet boundary condition, Advances in Water Resources, 34(3), 365 - 374, doi:http://dx.doi.org/10. 1016/j.advwatres.2010.12.008.

[11] Chiogna, G., C. Eberhardt, P. Grathwohl, O. A. Cirpka, and M. Rolle (2010), Evidence of compound-dependent hydrodynamic and mechanical transverse dispersion by multitracer laboratory experiments, Environmental Science \& Technology, 44(2), 688-693, doi:10.1021/es9023964, pMID: 20020677.

[12] Chiogna, G., O. A. Cirpka, P. Grathwohl, and M. Rolle (2011), Relevance of local compound-specific transverse dispersion for conservative and reactive mixing in heterogeneous porous media, Water Resources Research, 47(7), n/a-n/a, doi:10.1029/2010WR010270, w07540.

[13] Cirpka, O. A., and P. K. Kitanidis (2000), Characterization of mixing and dilution in heterogeneous aquifers by means of local temporal moments, Water Resources Research, 36(5), 1221-1236, doi:10.1029/1999WR900354.

[14] Cirpka, O. A., E. O. Frind, and R. Helmig (1999), Numerical simulation of biodegradation controlled by transverse mixing, Journal of Contaminant 
Hydrology, 40(2), 159 - 182, doi:http://dx.doi.org/10.1016/S0169-7722(99) 00044-3.

[15] Cirpka, O. A., Å. Olsson, Q. Ju, M. Rahman, P. Grathwohl, et al. (2006), Determination of transverse dispersion coefficients from reactive plume lengths, Ground Water, 44(2), 212-221.

[16] Cirpka, O. A., G. Chiogna, M. Rolle, and A. Bellin (2015), Transverse mixing in three-dimensional nonstationary anisotropic heterogeneous porous media, Water Resources Research, 51(1), 241-260, doi:10.1002/ 2014WR015331.

[17] Delgado, J. (2007), Longitudinal and transverse dispersion in porous media, Chemical Engineering Research and Design, 85(9), 1245-1252.

[18] Dentz, M., H. Kinzelbach, S. Attinger, and W. Kinzelbach (2002), Temporal behavior of a solute cloud in a heterogeneous porous medium 3. numerical simulations, Water Resources Research, 38(7), 23-1-23-13, doi: 10.1029/2001WR000436.

[19] Dentz, M., H. Kinzelbach, S. Attinger, and W. Kinzelbach (2003), Numerical studies of the transport behavior of a passive solute in a twodimensional incompressible random flow field, Phys. Rev. E, 67, 046,306, doi:10.1103/PhysRevE.67.046306.

[20] Dentz, M., T. L. Borgne, A. Englert, and B. Bijeljic (2011), Mixing, spreading and reaction in heterogeneous media: A brief review, Journal of Contaminant Hydrology, 120121(0), 1 - 17, doi:http://dx.doi.org/10.1016/j. jconhyd.2010.05.002, reactive Transport in the Subsurface: Mixing, Spreading and Reaction in Heterogeneous Media.

[21] Eberhardt, C., and P. Grathwohl (2002), Time scales of organic contaminant dissolution from complex source zones: coal tar pools vs. blobs, Journal of Contaminant Hydrology, 59(1), 45-66. 
[22] Frippiat, C. C., P. C. Perez, and A. E. Holeyman (2008), Estimation of laboratory-scale dispersivities using an annulus-and-core device, Journal of Hydrology, 362(1 - 2), 57 - 68, doi:http://dx.doi.org/10.1016/j.jhydrol. 2008.08.007.

[23] Gelhar, L. W., and C. L. Axness (1983), Three-dimensional stochastic analysis of macrodispersion in aquifers, Water Resources Research, 19(1), 161180, doi:10.1029/WR019i001p00161.

[24] Grane, F. (1961), Measurements of transverse dispersion in granular media., Journal of Chemical \&s Engineering Data, 6(2), 283-287, doi: 10.1021/je60010a031.

[25] Han, N.-W., J. Bhakta, and R. G. Carbonell (1985), Longitudinal and lateral dispersion in packed beds: Effect of column length and particle size distribution, AIChE Journal, 31(2), 277-288, doi:10.1002/aic.690310215.

[26] Harleman, D. R. F., and R. R. Rumer (1963), Longitudinal and lateral dispersion in an isotropic porous medium, Journal of Fluid Mechanics, 16, 385-394, doi:10.1017/S0022112063000847.

[27] Hassinger, R., and D. Von Rosenberg (1968), A mathematical and experimental examination of transverse dispersion coefficients., Society of Petroleum Engineers, Journal 8(1), 195-204., doi:10.2118/1846-PA.

[28] Kitanidis, P., and P. McCarty (2012), Delivery and Mixing in the Subsurface: Processes and Design Principles for In Situ Remediation, SERDP 75 ESTCP Environmental Remediation Technology, Springer New York.

[29] Kitanidis, P. K. (1994), The concept of the dilution index, Water Resources Research, 30(7), 2011-2026, doi:10.1029/94WR00762.

[30] Klenk, I., and P. Grathwohl (2002), Transverse vertical dispersion in groundwater and the capillary fringe, Journal of Contaminant Hydrology, 58(1), 111-128. 
[31] Liedl, R., A. J. Valocchi, P. Dietrich, and P. Grathwohl (2005), Finiteness of steady state plumes, Water Resources Research, 41(12), n/a-n/a, doi: 10.1029/2005WR004000, w12501.

[32] Massabo, M., M., F. Catania, and O. Paladino (2007), A new method for laboratory estimation of the transverse dispersion coefficient, Ground Water, 45(3), 339-347, doi:10.1111/j.1745-6584.2007.00301.x.

[33] Mayer, K. U., E. O. Frind, and D. W. Blowes (2002), Multicomponent reactive transport modeling in variably saturated porous media using a generalized formulation for kinetically controlled reactions, Water Resources Research, 38(9), 13-1-13-21, doi:10.1029/2001WR000862.

[34] Olsson, A., and P. Grathwohl (2007), Transverse dispersion of non-reactive tracers in porous media: A new nonlinear relationship to predict dispersion coefficients, Journal of Contaminant Hydrology, 92(34), 149 - 161, doi: http://dx.doi.org/10.1016/j.jconhyd.2006.09.008.

[35] Perkins, T. K., and O. C. Johnston (1963), A review of diffusion and dispersion in porous media, Society of Petroleum Engineers Journal, 3, 70-84, doi:10.2118/480-PA.

[36] Pini, R., S. C. Krevor, and S. M. Benson (2012), Capillary pressure and heterogeneity for the $\mathrm{CO}_{2}$ /water system in sandstone rocks at reservoir conditions, Advances in Water Resources, 38(0), 48 - 59, doi:http://dx. doi.org/10.1016/j.advwatres.2011.12.007.

[37] Rabbani, A., and S. Jamshidi (2014), Specific surface and porosity relationship for sandstones for prediction of permeability, International Journal of Rock Mechanics and Mining Sciences, 71, 25 - 32, doi:http://dx.doi.org/ 10.1016/j.ijrmms.2014.06.013.

[38] Riaz, A., M. Hesse, H. A. Tchelepi, and F. M. Orr (2006), Onset of convection in a gravitationally unstable diffusive boundary layer in porous media, Journal of Fluid Mechanics, 548, 87-111, doi:10.1017/S0022112005007494. 
[39] Robbins, G. A. (1989), Methods for determining transverse dispersion coefficients of porous media in laboratory column experiments, Water Resources Research, 25(6), 1249-1258, doi:10.1029/WR025i006p01249.

[40] Rolle, M., D. Hochstetler, G. Chiogna, P. Kitanidis, and P. Grathwohl (2012), Experimental investigation and pore-scale modeling interpretation of compound-specific transverse dispersion in porous media, Transport in Porous Media, 93(3), 347-362, doi:10.1007/s11242-012-9953-8.

[41] Sahimi, M. (1995), Flow and transport in porous media and fractured rock: from classical methods to modern approaches, $\mathrm{VCH}$, Weinheim, New York.

[42] Sahimi, M., B. D. Hughes, L. Scriven, and H. T. Davis (1986), Dispersion in flow through porous media - 1. one-phase flow, Chemical Engineering Science, 41(8), 2103 - 2122, doi:http://dx.doi.org/10.1016/0009-2509(86) 87128-7.

[43] Seagren, E. A., B. E. Rittmann, and A. J. Valocchi (1999), An experimental investigation of napl pool dissolution enhancement by flushing, Journal of contaminant hydrology, 37(1), 111-137.

825 [44] Steefel, C. I., C. A. J. Appelo, B. Arora, D. Jacques, T. Kalbacher, O. Kolditz, V. Lagneau, P. C. Lichtner, K. U. Mayer, J. C. L. Meeussen, S. Molins, D. Moulton, H. Shao, J. Šimůnek, N. Spycher, S. B. Yabusaki, and G. T. Yeh (2014), Reactive transport codes for subsurface environmental simulation, Computational Geosciences, 19(3), 445-478, doi: $830 \quad 10.1007 / \mathrm{s} 10596-014-9443-\mathrm{x}$.

[45] Szulczewski, M. L., C. W. MacMinn, H. J. Herzog, and R. Juanes (2012), Lifetime of carbon capture and storage as a climate-change mitigation technology, Proceedings of the National Academy of Sciences, 109(14), 51855189, doi:10.1073/pnas.1115347109.

835 [46] Thierrin, J., and P. K. Kitanidis (1994), Solute dilution at the borden 
and cape cod groundwater tracer tests, Water Resources Research, 30(11), 2883-2890, doi:10.1029/94WR01983.

[47] Wexler, E.J. (1992), Analytical solutions for one-, two-, and threedimensional solute transport in ground-water flow systems with uniform flow, vol. 3, Applications of hydraulics, chapter B7. US Geological Survey, 1992.

[48] Xiao, Y., T. Xu, and K. Pruess (2009), The effects of gas-fluid-rock interactions on $\mathrm{CO}_{2}$ injection and storage: Insights from reactive transport modeling, Energy Procedia, 1(1), 1783 - 1790, doi:http://dx.doi.org/10.1016/j. egypro.2009.01.233, greenhouse Gas Control Technologies 9 Proceedings of the 9th International Conference on Greenhouse Gas Control Technologies (GHGT-9), 1620 November 2008, Washington DC, \{USA\}. 